\title{
High-resolution rotation curves of low surface brightness galaxies ${ }^{\star}$
}

\author{
W. J. G. de Blok ${ }^{1}$ and A. Bosma ${ }^{2}$ \\ 1 ATNF, CSIRO, PO Box 76, Epping NSW 1710, Australia \\ 2 Observatoire de Marseille, 2 place Le Verrier, 13248 Marseille Cedex 4, France \\ Received 18 July 2001 / Accepted 15 January 2002

\begin{abstract}
We present high-resolution rotation curves of a sample of 26 low surface brightness galaxies. From these curves we derive mass distributions using a variety of assumptions for the stellar mass-to-light ratio. We show that the predictions of current Cold Dark Matter models for the density profiles of dark matter halos are inconsistent with the observed curves. The latter indicate a core-dominated structure, rather than the theoretically preferred cuspy structure.
\end{abstract}

Key words. galaxies: structure - galaxies: kinematics and dynamics - galaxies: halos

\section{Introduction}

Despite much effort, it is still unclear to what extent rotation curves can give clues about the distribution of the visible and dark matter in bright spiral galaxies (e.g. Bosma 1999; Sellwood 1999). It is thought, however, that low surface brightness (LSB) galaxies and dwarf galaxies are dark matter dominated, and that therefore the analysis of their rotation curves can yield directly important information about the properties and distribution of their associated dark matter halos (de Blok \& McGaugh 1997; Verheijen 1997; Swaters 1999).

This implication has far-reaching consequences, as early results by e.g. Moore (1994) already showed. Predictions from cold dark matter (CDM) simulations were found to disagree with observations of rotation curves of several dwarf galaxies; the data indicating much less cuspy distributions of matter than the simulations. It was thought at the time that this problem could be solved once the effect of feedback due to star formation was understood. However, the behaviour of the rotation curves of low surface brightness (LSB) galaxies is rather similar, and their low star formation rate at present and in the past indicate that star formation in such galaxies might never have been important enough to modify their structure drastically.

Early work on LSB galaxies used 21-cm Hi line work to determine the rotation curves (e.g. van der Hulst et al. 1993; de Blok et al. 1996), and as such the results are

\footnotetext{
Send offprint requests to: W. J. G. de Blok, e-mail: edeblok@atnf.csiro.au

* based on observations at the Observatoire de Haute Provence.
}

likely to suffer from modest angular resolution effects (commonly called beam smearing). Even though these can be partly modelled, experience shows that direct measurements are preferable (cf. discussions in Bosma 1978; Begeman 1987); in particular, supplementary data in the optical emission lines, such as $\mathrm{H} \alpha$ and [NII] are always useful (cf. Van der Kruit \& Bosma 1978; Rubin et al. 1989; Corradi et al. 1991; Swaters et al. 2000). Thus a whole industry has sprung up to combine optical and 21-cm line rotation curves of all sorts of gas rich galaxies.

For the particular problem of the dark matter distribution in LSB galaxies Swaters et al. (2000) presented supplementary $\mathrm{H} \alpha$ data for five LSB galaxies already observed in Hi by de Blok et al. (1996), and concluded that the influence of beam smearing on the Hi curves was severe enough to question earlier conclusions regarding dark matter content and rotation curve shape of LSB galaxies. McGaugh et al. (2001) and de Blok et al. (2001a) (hereafter dBMR) reanalysed these data, and concluded that the discrepancy between $\mathrm{H} \alpha$ and $\mathrm{HI}$ data is only really significant for one of these five galaxies. Conclusions regarding the dark matter content and the shape of the dark matter distribution in LSB galaxies are thus not affected.

In a comparison of pseudo-isothermal and CDM halo models using high-resolution rotation curves of a sample of a further 29 LSB galaxies dBMR show that the so-called "universal" CDM halo-profile as parameterised in Navarro et al. (1996) is not a good description of the data: the rotation curves generally show linear solid-body rise in the inner parts, which is inconsistent with the steeper rise necessary for the CDM rotation curves. Rather, the rotation curves prefer a pseudo-isothermal (i.e. core-dominated) halo model. de Blok et al. (2001b) furthermore showed that all rotation curves of LSB galaxies measured so 
far are consistent with a pseudo-isothermal core model. Analyses that show that these rotation curves confirm the CDM NFW halo model (e.g. van den Bosch \& Swaters 2001) can be traced back to the fact that at lower resolutions the NFW and pseudo-isothermal models look sufficiently similar and the errors are large enough that a NFW model can be made to fit the data. The higher-resolution data presented in de Blok et al. (2001b) now seem to have settled the observational side of the debate in favour of core-dominated LSB galaxy halos. These results are independent of the stellar mass-to-light ratios one assumes in constructing these mass models.

It would therefore seem that current models of structure formation and galaxy evolution need to take into account the fact that most late-type galaxies have a constant density dark matter core rather than a cusp (see also results by Borriello \& Salucci 2001; Salucci \& Burkert 2000; Salucci 2001 for HSB galaxies).

As the signature of the core is clearest at small radii it is important to find galaxies where these inner parts are well-resolved and well-sampled. In this paper we thus supplement the collection of high-resolution rotation curves of LSB galaxies from de Blok et al. (2001a) with curves for an additional 26 galaxies, of which 12 are entirely new and 14 were already used in the analysis by de Blok et al. (2001b). The 12 new galaxies in our sample have been specifically chosen to have small distances so that we can easily verify the discrepancies between the NFW model and the observations.

This paper is organised as follows: we describe our data in Sect. 2, and present the rotation curves in Sect. 3. Section 4 describes individual galaxies. In Sect. 5 we describe the derivation of the final rotation curves. The mass models are then presented in Sect. 6 . In Sect. 7 we discuss these models. Section 8 digresses into the consequences that systematic observational effects may or may not have on the data. In Sect. 9 we discuss the mass densities profiles inferred from the rotation curves. Section 10 summarises the paper.

\section{Observations and data reduction}

\subsection{Sample selection}

We constructed a sample of galaxies to observe in $\mathrm{H} \alpha$ from lists of LSB galaxies already observed in the 21-cm Hi line by van der Hulst et al. (1993) and de Blok et al. (1996). A representative sample of nearby dwarf galaxies from the theses of Swaters (1999) and Stil (1999) was also included (see also Swaters et al. 2002a,b). The sample was supplemented by two well-known nearby dwarf galaxies (NGC 1560 and NGC 100) and a galaxy (UGC 711) from the Flat Galaxy Catalog (Karachentsev et al. 1993). HI velocity fields were available for all galaxies (except U711 and N100). Since we observed with a long-slit spectrograph, we determined for each galaxy the position angle of the major axis of the velocity field, using the position angle of the major axis of the light distribution as an ad- ditional check. For U711 and N100 we only used the position angle of the light distribution: since these are edge-on galaxies these angles are well determined.

Table 1 lists parameters of these galaxies for which we managed to obtain useful, high $\mathrm{S} / \mathrm{N}$ data that will be used in our mass modelling. The last column in Table 1 gives references to the data presented in Table 1 not derived in this paper. Images and surface brightness profiles can also be found in these references. See also Sect. 2.4. Table 2 gives a brief summary of those galaxies that we did observe, but which for various reasons were deemed to be of insufficient quality to warrant mass modelling. Our main sample therefore consists of the galaxies listed in Table 1.

\subsection{Optical spectra}

The emission line observations have been made with the 193-cm telescope at the Observatoire de Haute Provence and its long-slit Carelec spectrograph from 2-9 Jan. 2000 and 22-27 Feb. 2001. The spectrograph has been described in detail by Lemaître et al. (1991). As a detector, we used a EEV $2048 \times 1024$ CCD chip. The slit length on the sky was about $5.5^{\prime}$, and we used a $2.0^{\prime \prime}$ slit-width. This works out to a pixel size of $0.6^{\prime \prime} \times 45.7 \mathrm{~km} \mathrm{~s}^{-1}$. Spectral resolution was $54 \mathrm{~km} \mathrm{~s}^{-1}$ FWHM. Typical exposure times were 1 hour per spectrum, preceded and followed by calibration spectra from a neon arc lamp. The typical seeing during the observations was $\sim 2^{\prime \prime}$. Care was taken to align the slit with the optical centre of the galaxy. This was done with off-set pointing from nearby stars. In many cases the central part of the galaxy was visible in the guiding camera, and the accuracy of the off-set procedure could be verified. For a few of these galaxies we repeated the off-set procedure a few times, and found that the galaxy acquisition was indeed repeatable, with an error of less than $1^{\prime \prime}$. See Sect. 8 for a full discussion of the effects of (mis-)pointing.

\subsection{Data reduction}

The data were corrected for instrumental effects (bias, flatfield, etc.) in the standard manner. The spectra were then further reduced with the Figaro package: the calibration spectra were averaged, and for each of the spectra a wavelength solution was found and the wavelength calibration applied. Cosmic rays were corrected with an automated method, which was verified interactively.

The data were then further processed using the EMSAO programme of the RVSAO package (Kurtz \& Mink 1998) within the IRAF environment. Emission lines were traced and their wavelengths determined using known night sky lines. Spectral shifts were then converted into barycentric radial velocities. The spectra were averaged every 3 pixels in spatial direction $\left(1.8^{\prime \prime}\right)$. Some spectra with low $S / N$ were averaged every 9 pixels $\left(5.4^{\prime \prime}\right)$ as indicated in Table 1. 
Table 1. Properties of sample galaxies.

\begin{tabular}{|c|c|c|c|c|c|c|c|c|c|c|c|c|c|c|}
\hline $\begin{array}{l}\text { UGC } \\
(1)\end{array}$ & $\begin{array}{l}\text { Other } \\
(2)\end{array}$ & $\begin{array}{r}D \\
(3)\end{array}$ & $\begin{array}{l}M_{R} \\
(4)\end{array}$ & $\begin{array}{l}\mu_{0, R} \\
(5)\end{array}$ & $\begin{array}{l}h_{R} \\
(6)\end{array}$ & $\begin{array}{c}i \\
(7)\end{array}$ & $\begin{array}{r}R_{\max } \\
(8)\end{array}$ & $\begin{array}{r}V_{\max } \\
(9)\end{array}$ & $\begin{array}{l}V_{\text {sys }} \\
(10)\end{array}$ & $\begin{array}{r}V_{\mathrm{sys}, \mathrm{HI}} \\
(11)\end{array}$ & $\begin{array}{r}\mathrm{PA} \\
(12)\end{array}$ & $\begin{array}{l}\text { bin } \\
(13)\end{array}$ & $\begin{array}{l}\text { Obs. } \\
(14)\end{array}$ & $\begin{array}{l}\text { Refs. } \\
(15)\end{array}$ \\
\hline- & F563-1 & 45 & -18. & 22.6 & 3.5 & 25 & 17.5 & 114 & 3495 & 3492 & 161 & 5.4 & Jan. 00 & $\overline{(1)(2)}$ \\
\hline U231 & N100 & 11.2 & $-17.7^{a}$ & - & - & 89 & 8.3 & 97 & 841 & - & 236 & 1.8 & Jan. 00 & - \\
\hline U628 & - & 65 & -19.2 & 22.1 & 4.7 & 56 & 13.8 & 142 & 5451 & - & 139 & 1.8 & Jan. 00 & $(2)(4)$ \\
\hline U711 & - & 26.4 & $-17.7^{a}$ & - & - & 90 & 15.4 & 92 & 1984 & - & 118 & 1.8 & Jan. 00 & - \\
\hline U731 & DDO 9 & 8.0 & -16.6 & 23.0 & 1.7 & 57 & 7.0 & 75 & 637 & 638 & 257 & 1.8 & Jan. 00 & $(5)$ \\
\hline U1230 & - & 51 & -19.1 & 22.6 & 4.5 & 22 & 34.7 & 103 & 3837 & 3835 & 112 & 1.8 & Jan. 00 & $(2)(4)$ \\
\hline U1281 & - & 5.5 & -16.2 & 22.7 & 1.7 & 90 & 5.2 & 57 & 157 & 157 & 218 & 1.8 & Jan. 00 & $(5)$ \\
\hline U3060 & N1560 & 3.0 & $-15.9^{a}$ & $23.2^{a}$ & $1.3^{a}$ & 82 & 8.3 & 78 & -36 & -36 & 203 & 1.8 & Jan. 00 & $(3)$ \\
\hline U3137 & - & 18.4 & -18.7 & 23.2 & 2.0 & 90 & 30.9 & 100 & 982 & 993 & 255 & 1.8 & Jan. 00 & $(5)$ \\
\hline U3371 & DDO 39 & 12.8 & -17.7 & 23.3 & 3.1 & 49 & 10.3 & 86 & 819 & 818 & 133 & 5.4 & Jan. 00 & $(5)$ \\
\hline U4173 & - & 16.8 & -17.8 & 24.3 & 4.5 & 40 & 12.2 & 57 & 861 & 865 & 168 & 5.4 & Jan. 00 & $(5)$ \\
\hline U4325 & N2552 & 10.1 & -18.1 & 21.6 & 1.6 & 41 & 4.6 & 123 & 523 & 523 & 231 & 1.8 & Jan. 00 & $(5)$ \\
\hline U5005 & - & 52 & -18.6 & 22.9 & 4.4 & 41 & 27.7 & 99 & 3830 & 3844 & 226 & 5.4 & Jan. 00 & $(2)(4)$ \\
\hline U5750 & - & 56 & -19.5 & 22.6 & 5.6 & 64 & 21.8 & 79 & 4169 & 4168 & 167 & 1.8 & Jan. 00 & $(2)(4)$ \\
\hline U3851 & N2366 & 3.4 & -16.9 & 22.6 & 1.5 & 59 & 5.4 & 55 & 102 & 104 & 42 & 1.8 & Feb. 01 & (5) \\
\hline U3974 & DDO47 & $4^{b}$ & $-14.9^{a}$ & - & 1.0 & 30 & 3.2 & 67 & 282 & 274 & 319 & 5.4 & Feb. 01 & (6) \\
\hline U4278 & I 2233 & 10.5 & -17.7 & 22.5 & 2.3 & 90 & 7.4 & 93 & 556 & 559 & 173 & 1.8 & Feb. 01 & (5) \\
\hline U4426 & DDO52 & 5.3 & $-13.8^{a}$ & - & 0.6 & 60 & 3.1 & 50 & $382:$ & 395 & 185 & 1.8 & Feb. 01 & (6) \\
\hline U5272 & DDO64 & 6.1 & $-14.7^{a}$ & - & 1.2 & 60 & 2.7 & 47 & 520 & 525 & 97 & 1.8 & Feb. 01 & (6) \\
\hline U5721 & N3274 & 6.7 & -16.7 & 20.2 & 0.5 & 61 & 7.3 & 79 & 542 & 542 & 279 & 1.8 & Feb. 01 & $(5)$ \\
\hline U7524 & N4395 & 3.5 & -18.1 & 22.2 & 2.3 & 46 & 7.9 & 83 & 319 & 320 & 327 & 1.8 & Feb. 01 & (5) \\
\hline U7603 & N4455 & 6.8 & -16.9 & 20.8 & 0.7 & 78 & 5.9 & 64 & 655 & 644 & 197 & 1.8 & Feb. 01 & $(5)$ \\
\hline U8286 & N5023 & 4.8 & -17.2 & 20.9 & 0.8 & 90 & 5.9 & 84 & 403 & 407 & 208 & 1.8 & Feb. 01 & $(5)$ \\
\hline U8837 & DDO185 & 5.1 & -15.7 & 23.2 & 1.2 & 80 & 2.1 & 50 & 148 & 135 & 22 & 1.8 & Feb. 01 & (5) \\
\hline U9211 & DDO189 & 12.6 & -16.2 & 22.6 & 1.2 & 44 & 8.3 & 64 & 685 & 686 & 287 & 5.4 & Feb. 01 & (5) \\
\hline U10310 & Arp 2 & 15.6 & -17.9 & 22.0 & 1.9 & 34 & 9.0 & 75 & 720 & 718 & 202 & 1.8 & Feb. 01 & (5) \\
\hline
\end{tabular}

Explanation of columns: (1) UGC number (2) Other identification (3) Distance [Mpc] (see text) (4) Absolute magnitude in $R$-band corrected for Galactic extinction [mag]. (5) Central surface brightness in $R$-band, corrected for Galactic extinction and inclination [mag $\operatorname{arcsec}^{-2}$ ] (6) Scale length [kpc] (7) Inclination [ ${ }^{\circ}$ ] (8) Maximum radius rotation curve [kpc] (9) Maximum rotation velocity $\left[\mathrm{km} \mathrm{s}^{-1}\right]$ (10) Systemic velocity $\left[\mathrm{km} \mathrm{s}^{-1}\right]$ (11) Systemic velocity derived from Hi data [ $\left.\mathrm{km} \mathrm{s}^{-1}\right]$ (12) Position angle slit $\left[^{\circ}\right.$ ] (13) Binning interval rotation curve [arcsec] (14) Observing run (15) References: (1) de Blok et al. (1996) (2) de Blok et al. (1995) (3) Broeils (1992) (4) van der Hulst et al. (1993) (5) Swaters (1999) (6) Stil (1999). Notes: (a) B-band data. (b): distance from Walter \& Brinks (2001).

\subsection{Data collected from the literature}

Since our aim is to make mass models of our galaxies, we collected supplementary data from the literature, basically optical surface photometry and Hi data. The references are listed in the notes to Table 1 (see also Swaters et al. 2002a,b). For 22 out of the 26 galaxies studied here optical $R$-band ( $B$-band where noted in Table 1 ) is available, as well as $\mathrm{HI}$ imaging and $\mathrm{HI}$ and $\mathrm{H} \alpha$ rotation curves. Of these 22 galaxies, 5 are (close to) edge-on and because of the uncertainties involved in de-projecting surface brightness profiles of edge-on galaxies we will not consider their surface photometry here. See also Sect. 6.1.

For U628 we only have $R$-band photometry and $\mathrm{H} \alpha$ velocity data. For two galaxies (U711 and N100) we only have $\mathrm{H} \alpha$ data. For U10310 we only have $\mathrm{HI}$ and $\mathrm{H} \alpha$ data.
For these four galaxies we can construct only limited mass models.

Generally we adopted the distances given in the source papers (corrected to $H_{0}=75 \mathrm{~km} \mathrm{~s}^{-1} \mathrm{Mpc}^{-1}$ ), except for DDO 47 where we used the revised distance given in Walter \& Brinks (2001), and for U711 where we used the recession velocity. Inclinations were also adopted from the source papers. These inclinations are for the major part kinematically derived and as such only have a few degrees error at most. It should be emphasised that the shape of the rotation curve and hence applicability of any particular model does not depend on inclination, and thus an uncertainty of a few degrees does not affect our main conclusions. The scale lengths and surface brightnesses were are also taken from the references listed in Table 1 . 
W. J. G. de Blok and A. Bosma: LSB galaxy rotation curves
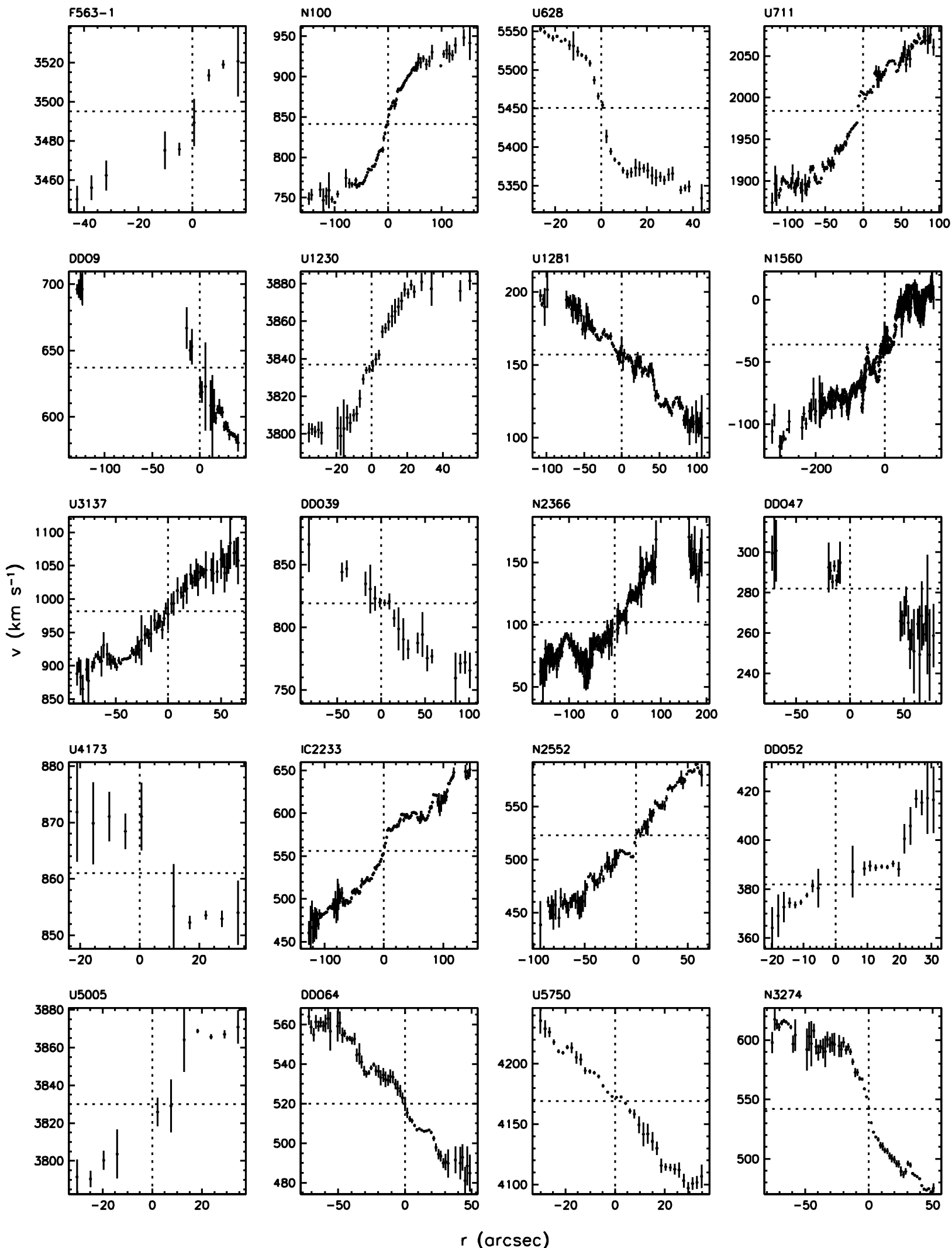

Fig. 1. Raw $\mathrm{H} \alpha$ velocity curves. The centre and systemic velocity are indicated by dotted lines.

We refer to these papers for precise details. Here it suffices to say that they were derived using a simple straight-line fit to the exponential part of the profile (most of the sample galaxies are dominated by an exponential disk).

\section{Results}

\subsection{Raw $\mathrm{HI}$ and $\mathrm{H} \alpha$ data}

Our principal results are collected in Figs. 1 to 5. Figure 1 shows the raw velocity curves derived from the $\mathrm{H} \alpha$ data 

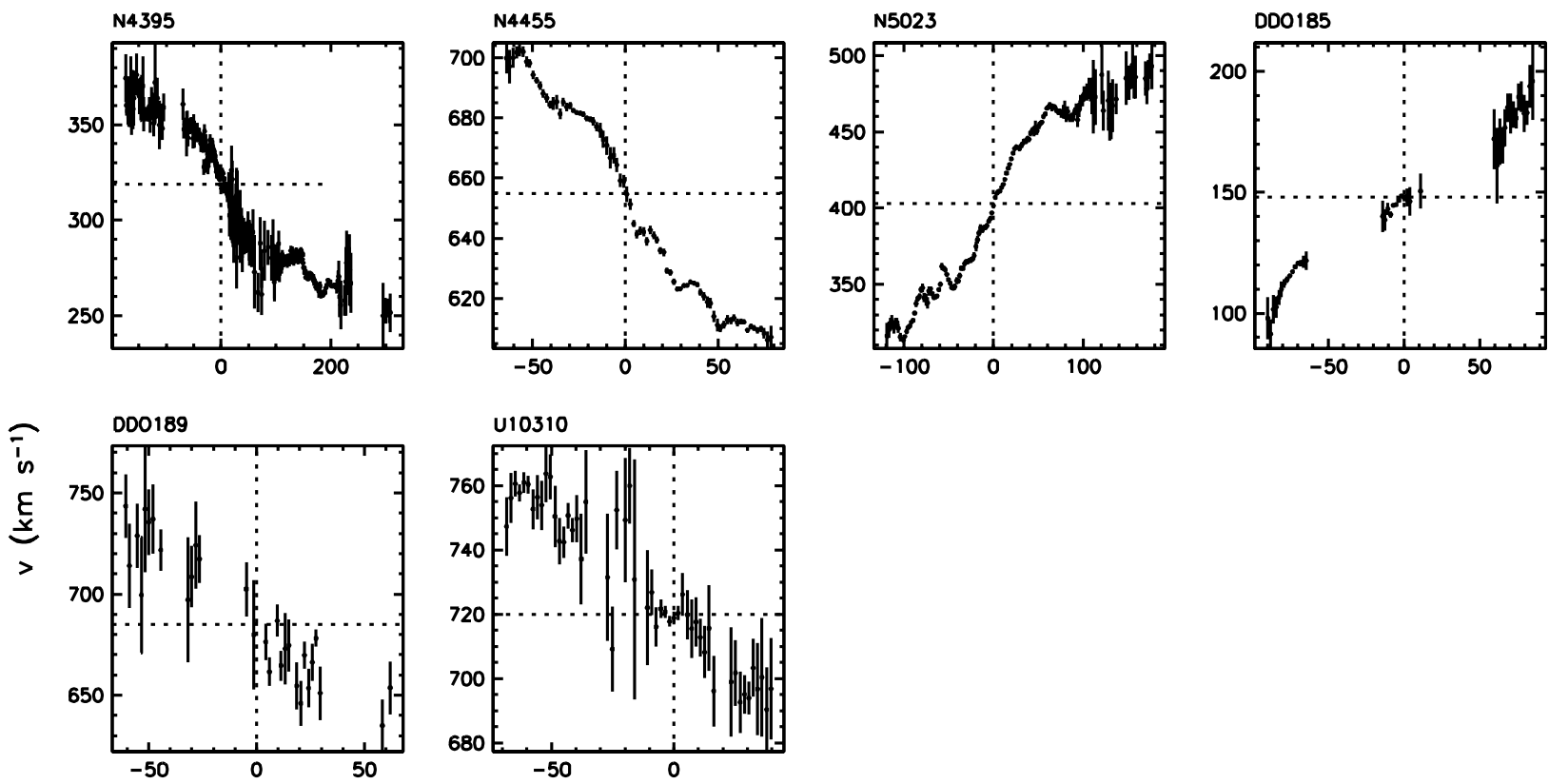

$r(\operatorname{arcsec})$

Fig. 1. continued.

Table 2. Galaxies not analysed.

\begin{tabular}{lc}
\hline Name & Reason for rejection \\
\hline Jan 2000 & observations \\
F561-1 & low S/N \\
F564-V3 & no H $\alpha$ detected \\
F568-6 & only bulge visible \\
U5999 & suspect slit position \\
DDO154 & low S/N, suspect slit position \\
DDO127 & low S/N \\
\hline Feb 2001 & observations \\
N4214 & suspect slit position \\
DDO125 & low S/N \\
DDO43 & low S/N \\
DDO168 & low S/N \\
\hline
\end{tabular}

for the galaxies listed in Table 1 . Figure 2 shows the raw data of the galaxies listed in Table 2. These latter data will not be analysed further.

Figure 3 presents overlays of the $\mathrm{H} \alpha$ data on a major axis position-velocity diagram from the Hi data. Such a presentation has the advantage of immediately showing any discrepancies between the two datasets, if they exist. The Hi data are taken from the literature, with references given in Table 1. Also indicated is the Hi rotation curve as derived from the position-velocity diagram. These overlays will be discussed in more detail in Sect. 4 .

\subsection{Symmetrised rotation curves}

In most cases the continuum emission from the central parts of the galaxies was visible in the spectra. This was used to determine the central position in the spectra and the systemic velocities. In a few cases (DDO185, U10310, U5999) the centre was not seen and we varied the central position and systemic velocity over a small range until we found the values that gave the maximum symmetry. The systemic velocities based on the $\mathrm{H} \alpha$ data are given in Table 1. A comparison with the systemic velocities derived from the Hi data (also listed in Table 1 and taken from the source papers) shows a good correspondence with the $\mathrm{H} \alpha$ velocities. The absolute difference is $5 \mathrm{~km} \mathrm{~s}^{-1}$ or less, except in 6 cases where the difference lies between 10 and $14 \mathrm{~km} \mathrm{~s}^{-1}$. In 4 of the 6 cases the Hi data were obtained using a velocity resolution of over $20 \mathrm{~km} \mathrm{~s}^{-1}$, implying that the comparison with $\mathrm{H} \alpha$ is still very favourable. Only for DDO 47 and DDO 52 do we find a difference of $\sim 1.5 \mathrm{HI}$ channel widths. Given the limited spatial and velocity resolution of the Hi data it is not clear where this difference originates.

Figure 4 shows the folded and symmetrised rotation curves, corrected for inclination.

Figure 5 compares the folded $\mathrm{H} \alpha$ rotation curves with the Hi rotation curves as presented in the literature (see Table 1 for references). It is clear that grosso modo there is reasonable agreement. A small number of galaxies were obviously affected by beam-smearing, just as for some galaxies the $\mathrm{H} \alpha$ rotation curve shows signs of non-circular motion. 

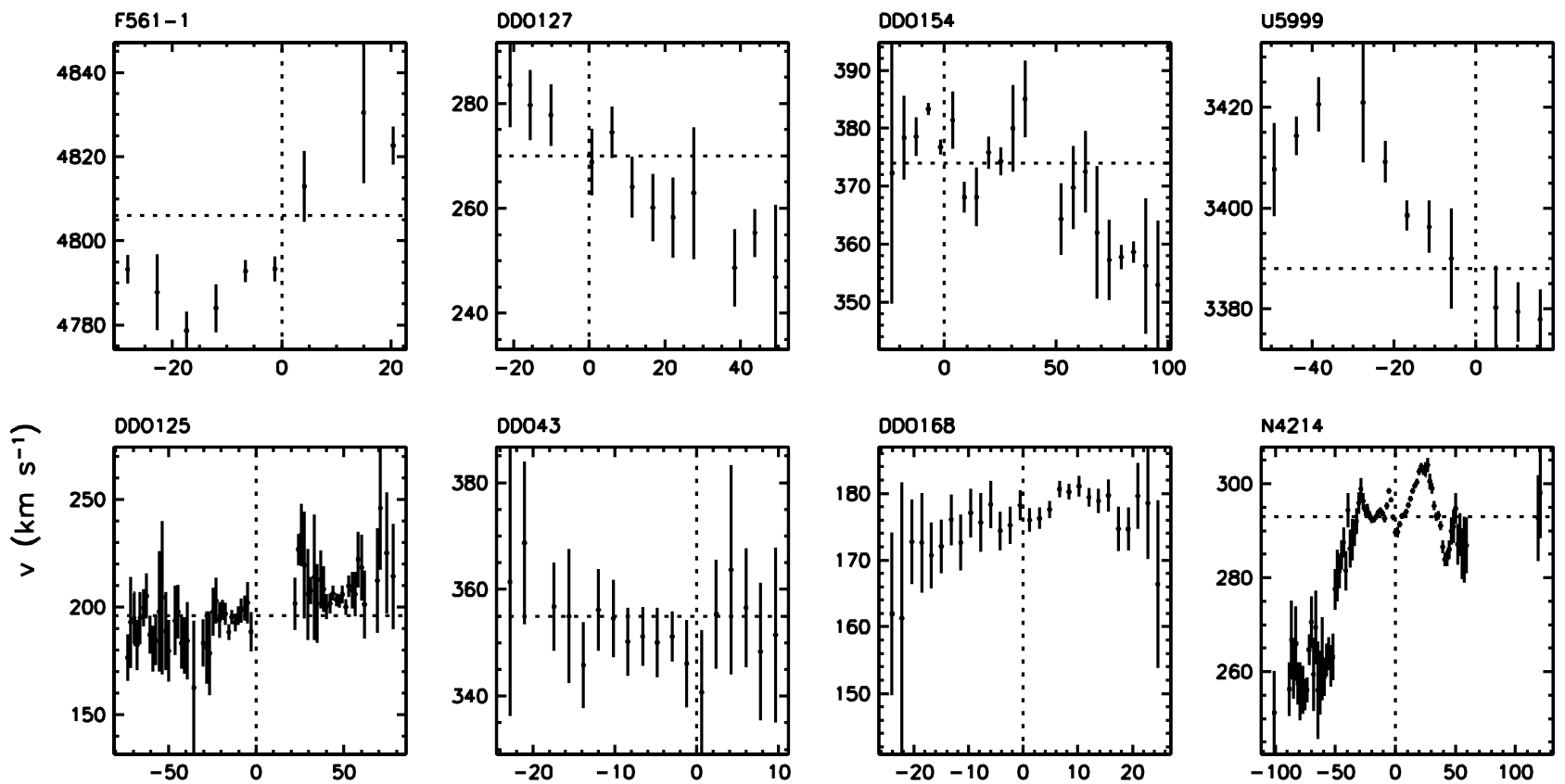

\section{$r(\operatorname{arcsec})$}

Fig. 2. Raw $\mathrm{H} \alpha$ velocity curves of galaxies not used in modelling. The centre and systemic velocity are indicated by dotted lines.

\section{Description of individual galaxies}

In this section we describe the various rotation curves of each galaxy in some detail. We will compare the Hi and the $\mathrm{H} \alpha$ data, as well as the $\mathrm{HI}$ and $\mathrm{H} \alpha$ rotation curves as presented in Figs. 1 and 3-5. For some galaxies we make a comparison with independent data from the literature.

F563-1: The $\mathrm{H} \alpha$ data show reasonable agreement with the HI data, showing that beamsmearing is not a major problem. At the receding side the $\mathrm{H} \alpha$ data disagree slightly with the Hi curve. An independent observation by dBMR of this galaxy is available and agrees with our observation (see Fig. 6); any systematic differences between the data sets is less than the uncertainties in individual data points. Obtaining high-resolution $\mathrm{H} \alpha$ rotation curves of LSB galaxies is thus a repeatable exercise.

U5005: The $\mathrm{H} \alpha$ data agree with the HI positionvelocity diagram, but the resulting high-resolution curve rises less steeply than the Hi curve. The Hi data have only a low spatial resolution of $40^{\prime \prime}$ (van der Hulst et al. 1993) and uncertain corrections for beam smearing are thus unavoidable.

U1230: The $\mathrm{H} \alpha$ data are of high-quality, and shows that beamsmearing has affected the $40^{\prime \prime}$-resolution $\mathrm{HI}$ curve (van der Hulst et al. 1993). The high-resolution curve rises more steeply, though still with a solid-body signature.

U5750: The Hi data are poor, but in agreement with the $\mathrm{H} \alpha$ data. The linearly rising Hi curve also shows reasonable agreement with the $\mathrm{H} \alpha$ data. For this galaxy an independent observation of dBMR is available and shows excellent agreement (Fig. 6), again showing that systematic effects due to telescope pointing etc. are negligible.

U731/DDO9: The $\mathrm{H} \alpha$ is weak at the receding side and superimposed on a night sky line. This makes it virtually impossible to say anything about the $\mathrm{H} \alpha$ velocities between $+0.5^{\prime}$ and $+2^{\prime}$. The $\mathrm{H} \alpha$ is stronger at the approaching side, and agrees well with the ridge in the HI $p V$ diagram. The $\mathrm{H} \alpha$ curve rises more steeply than the HI curve, which turns over too quickly. U731 is a "kinematically lopsided" galaxy (Swaters et al. 1999), and the HI rotation curve of the approaching side is different from that of the receding side. The $\mathrm{H} \alpha$ data agrees well with the steep approaching side of the Hi position-velocity diagram. The $\mathrm{H} \alpha$ curve is however significantly steeper than the Hi curve. This discrepancy cannot be explained by lopsidedness effects, which are much smaller than the difference shown here. The Hi curve extends over $\sim 8$ beams in radius, and this galaxy thus demonstrates that even for well-resolved galaxies corrections for beam-smearing are ambiguous, and do not always yield the correct outcome.

U1281, U3137, U3371/DDO39, U4325/N2552: For these four galaxies there is excellent agreement between the Hi position velocity data and the $\mathrm{H} \alpha$ curve. There is some disagreement between the $\mathrm{HI}$ and $\mathrm{H} \alpha$ rotation curves for U4325 and U3371. In these cases the HI curves rise more steeply than the $\mathrm{H} \alpha$ data. The agreement between the raw $\mathrm{HI}$ and $\mathrm{H} \alpha$ data suggests that the cause of this disagreement is likely an overcorrection for beamsmearing in the Hi data. While suited for 

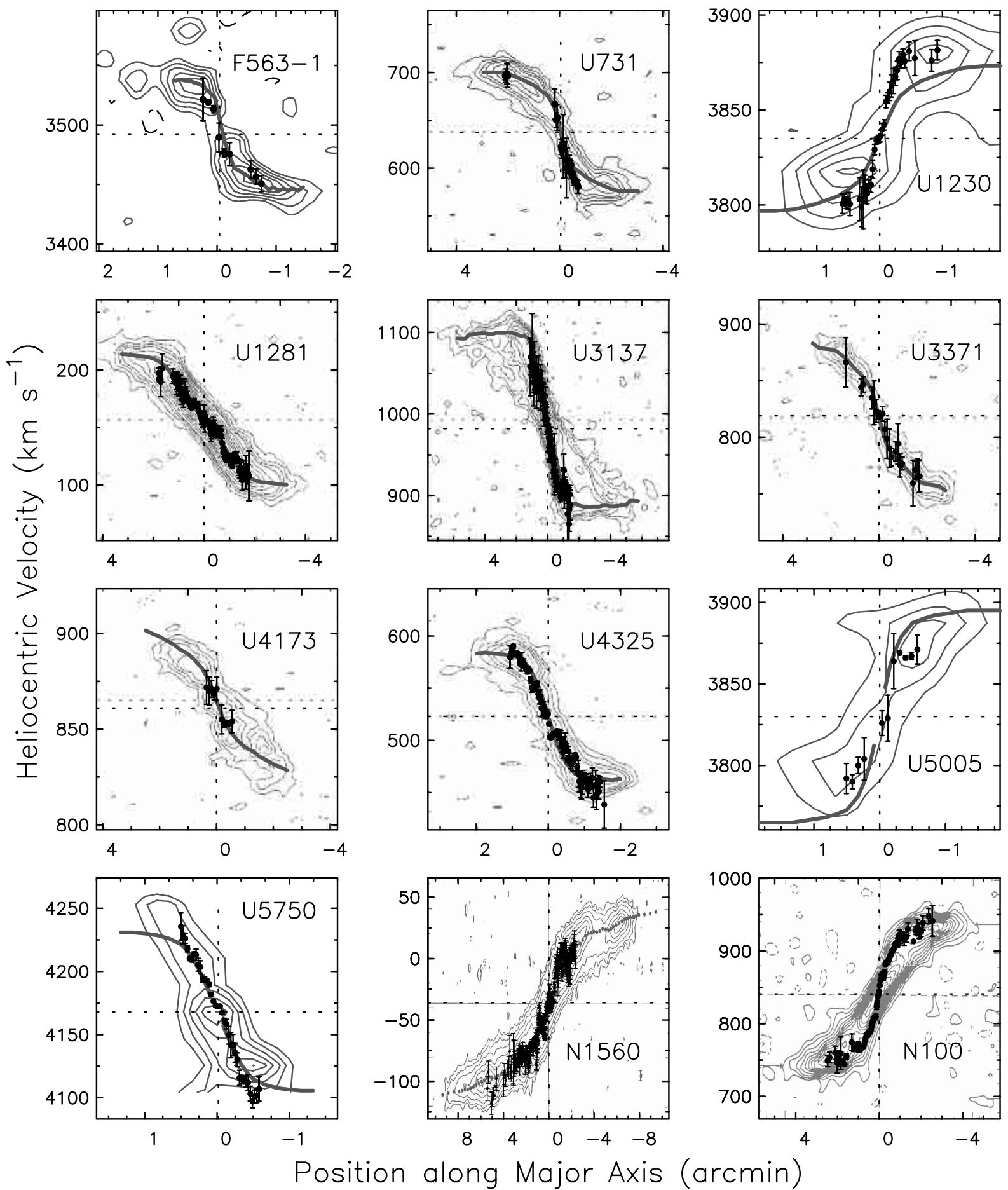

Fig. 3. Overlay of the raw $\mathrm{H} \alpha$ data on Hi position velocity diagrams. See Table 1 for the source of the Hi data. The H $\alpha$ data are indicated by the black dots. The rotation curves based on $\mathrm{HI}$ data are indicated by a dark-grey line.

correcting for large-scale resolution effects, beam smearing corrections thus give non-unique solutions on small scales - a rotation curve that looks like it has linear rise, can in reality be a steeply rising rotation curve (as the beam-smearing corrections in this case usually assume), or it can be an intrinsically linearly rising curve, as the $\mathrm{H} \alpha$ data in this case show.
U4173: The $\mathrm{H} \alpha$ data are rather poor in quality, but do seem to agree with the Hi data.

N100/U231: Good agreement between the data sets. Given the edge-on orientation of the galaxy, it reaffirms the transparency of this small galaxy, cf. Bosma et al. (1992). 


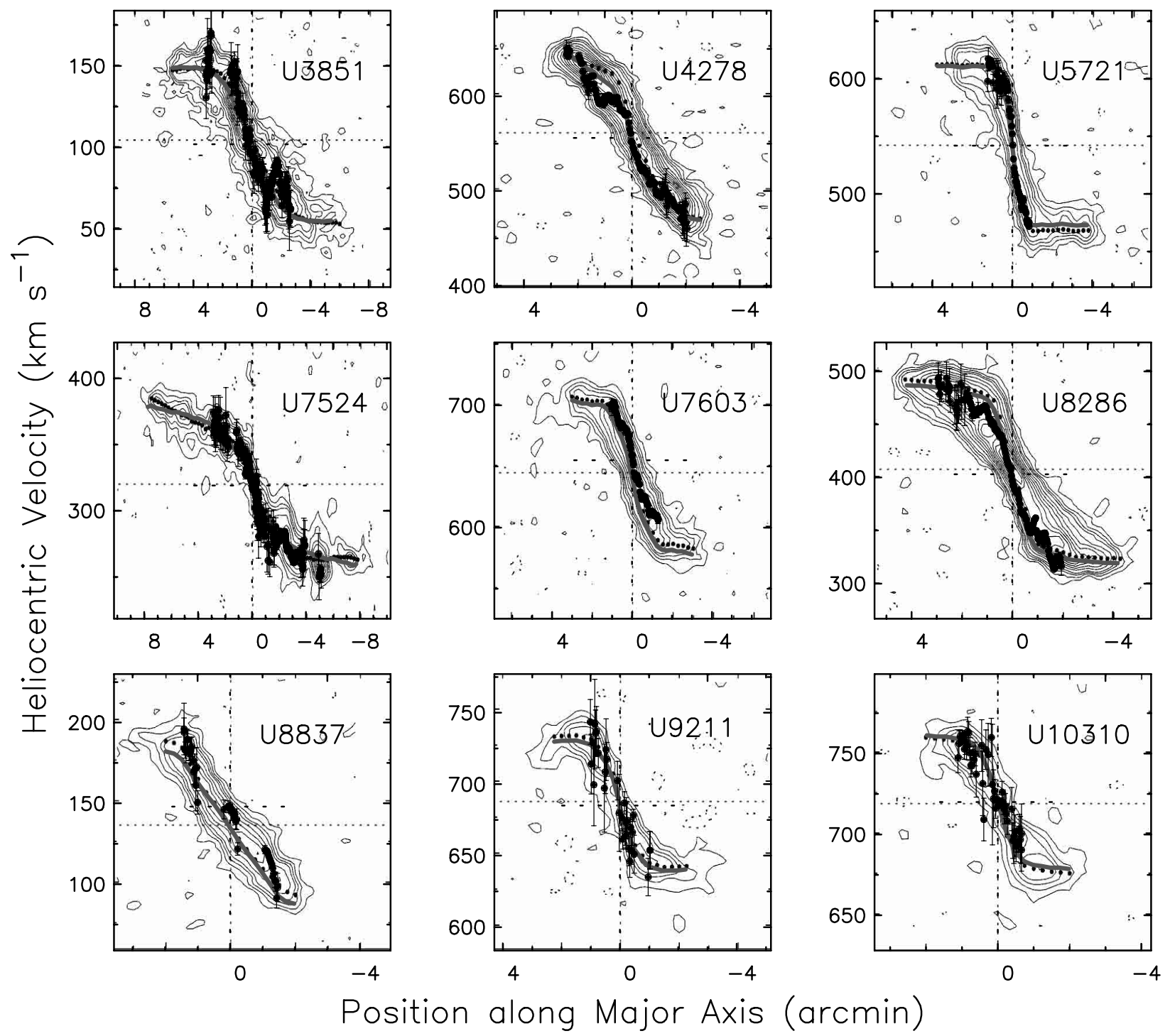

Fig. 3. continued.

N1560/U3060: Excellent agreement between HI and $\mathrm{H} \alpha$. This curve is a combination of three spectra covering the entire extent of the optical disk of N1560. The $\mathrm{H} \alpha$ curve shows clear signs of non-circular motions in the disk of N1560 (high-velocity gas near star forming regions, etc.).

N2366/U3851/DDO42: The $\mathrm{H} \alpha$ rotation-curve is dominated at the approaching side by the effects of noncircular motions. This position corresponds to a large star forming region (Hunter et al. 2001), that is disturbing the dynamics on that side of the galaxy. The unaffected raw $\mathrm{H} \alpha$ data show excellent agreement with the Hi positionvelocity diagram, the $\mathrm{H} \alpha$ rotation curve, however, rises steeper than the Hi curve.

N3274/U5721, N4395/U7524: In general there is excellent agreement between the various raw data sets. The $\mathrm{H} \alpha$ curves rise a bit faster than the Hi curves.

N4455/U7603: The H $\alpha$ agrees well with the Hi position velocity diagram. The Hi rotation curve seems to over-estimate the rotation velocity in the outer parts by a significant amount. For this galaxy we found a $\sim 10 \mathrm{~km} \mathrm{~s}^{-1}$ difference between the systemic velocities of the $\mathrm{HI}$ and $\mathrm{H} \alpha$ curves. The velocity resolution of the HI data is, however, $25 \mathrm{~km} \mathrm{~s}^{-1}$, so this difference may not be significant.

N5023/U8286: Excellent agreement between the data sets, except between $\sim 50^{\prime \prime}$ and $\sim 100^{\prime \prime}$ where the HI curve overestimates the velocity. The good agreement in the inner parts of this edge-on galaxy confirm again that these late-type galaxies are virtually transparent.

U10310/Arp2: The $\mathrm{H} \alpha$ data of this galaxy is rather poorer in quality than the previous couple of data sets. Nevertheless the data sets are in good agreement, except perhaps the Hi rotation curve which seems to overestimate the true rotation velocity.

DDO47/U3974: The $\mathrm{H} \alpha$ curve is rather poor in quality, but shows reasonable agreement with the Hi curve.

DDO52/U4426: Rather poor $\mathrm{H} \alpha$ data but a reasonable match with the Hi curve, except in the outer parts, where beam smearing effects presumable cause a small discrepancy. 


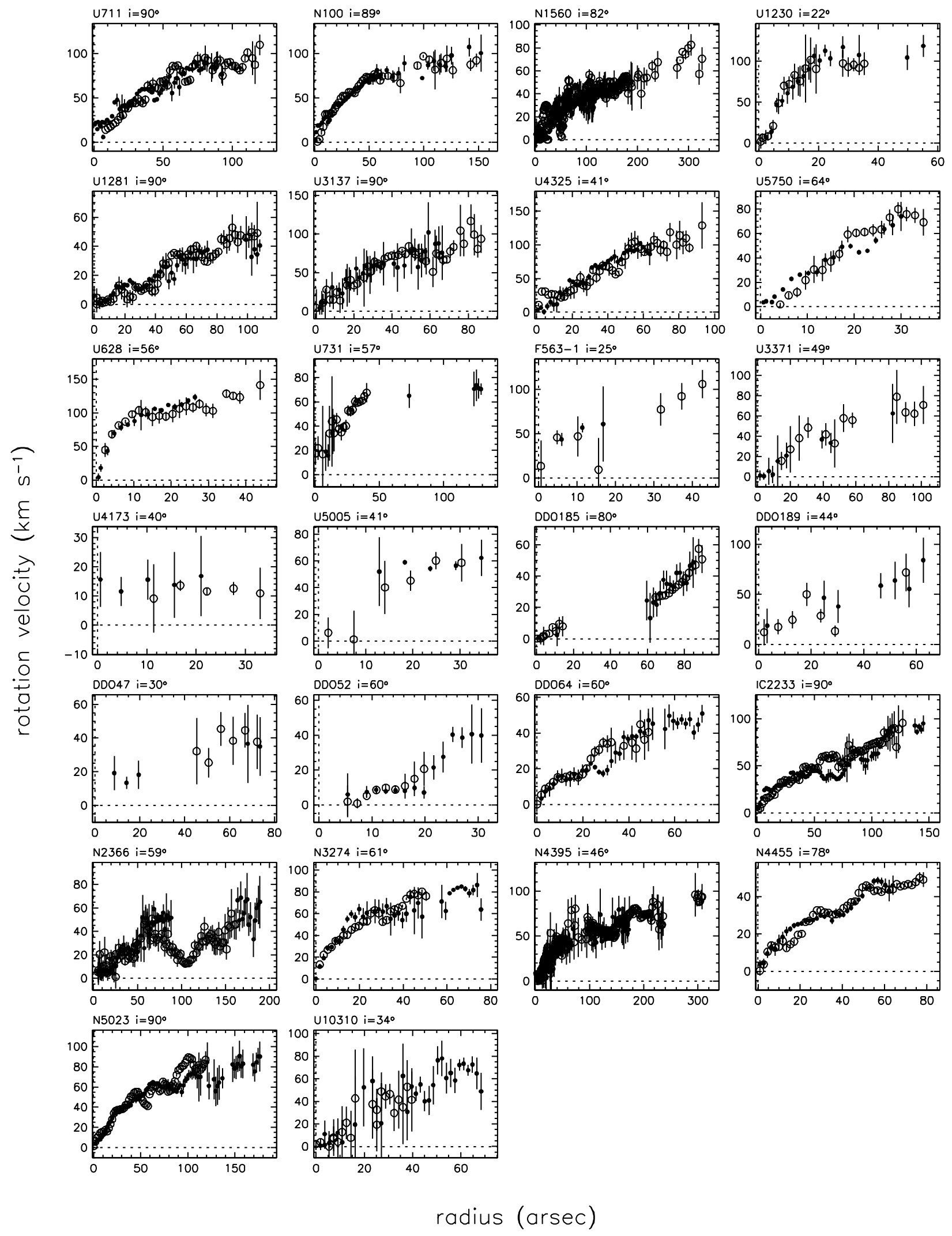

Fig. 4. Symmetrised and folded rotation curves. Different symbols indicate different sides of the galaxies. The rotation velocities have been corrected for inclination. 

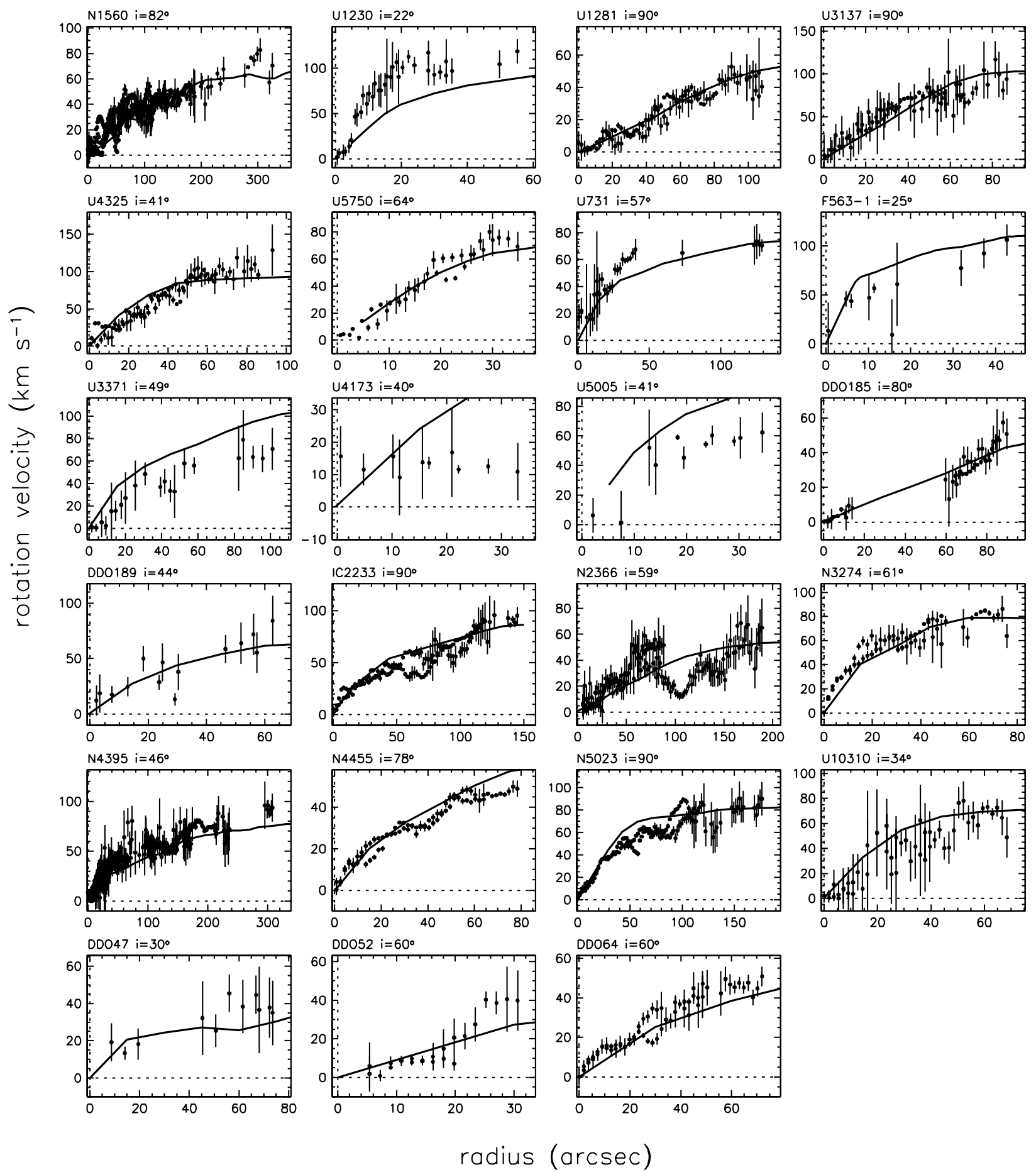

radius (arcsec)

Fig. 5. Comparison of the folded $\mathrm{H} \alpha$ rotation curves (black dots) with the Hi rotation curve (heavy line).

DDO64/U5272: Good $\mathrm{H} \alpha$ data show that the HI curve suffers from slight beam smearing effects. Noncircular motions presumably cause the difference between approaching and receding sides at $r \sim 30^{\prime \prime}$.

DDO185/U8837: The $\mathrm{H} \alpha$ data are of good quality, but do not correspond very well with the Hi positionvelocity overlay nor the Hi curve. The most likely explanation is non-circular motions in especially the approaching side, where the DSS image shows what is likely a large star formation complex. The receding side does show a linear rise. We do find an $\mathrm{H} \alpha$ systemic velocity that differs $\sim 15 \mathrm{~km} \mathrm{~s}^{-1}$ from the Hi systemic velocity. Alternatively, as this is one of the galaxies where the continuum emission of the central part could not be detected, we may have chosen the wrong systemic velocity. This curve is therefore rather uncertain.

DDO189/U9211: The $\mathrm{H} \alpha$ data are of average quality. The various data sets are all consistent with each other. 


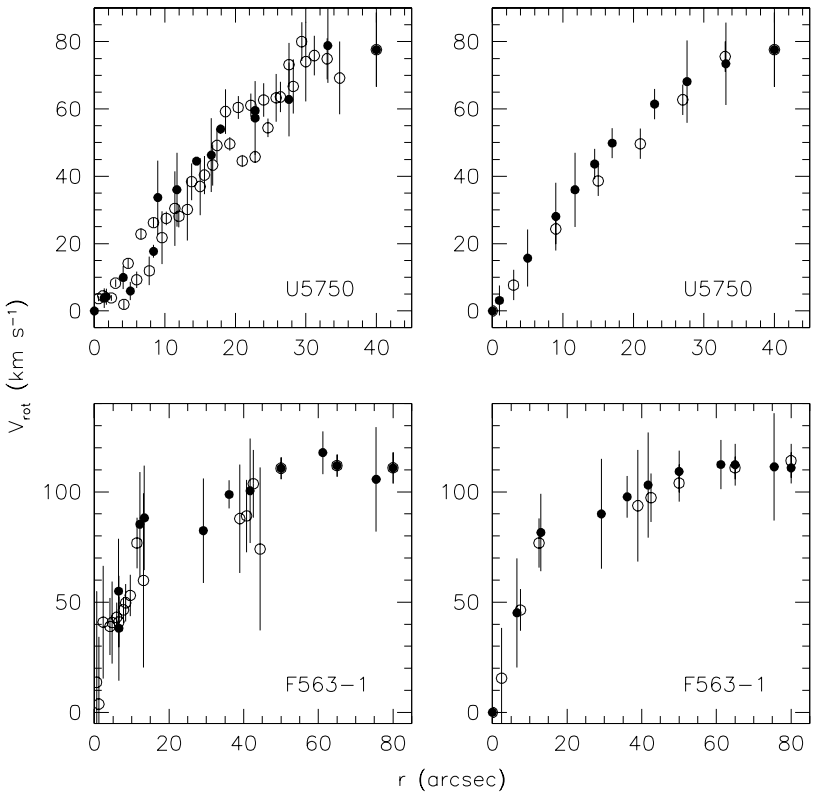

Fig. 6. Comparison of the data obtained by dBMR (open circles) and our data (filled circles), for two galaxies in common. At left is the folded original data, and at right the final adopted smooth rotation curves. The agreement is reasonable for UGC 5750 and F563-1.

In summary the comparison between $\mathrm{HI}$ and $\mathrm{H} \alpha$ shows that the Hi curves in this sample tend to suffer from two systematic effects. The low-resolution curves clearly suffer from beam-smearing which underestimates the rotation velocities. This is apparent in e.g. U1230. A second effect is apparent for a small number of galaxies where the raw $\mathrm{HI}$ and $\mathrm{H} \alpha$ data are an excellent match, but where the Hi curve overestimates the true rotation velocity. Because of the good match between the raw data sets, this systematic effect must have been introduced as a result of beam-smearing corrections; U4325 is a good example.

This comparison underscores the need for this kind of high-resolution $\mathrm{H} \alpha$ data even in moderately well-resolved galaxies. Beam-smearing corrections are useful in deriving the general properties of rotation curves. For example, they can tell us what the general shape of the original curve was, or how likely it is that a particular rotation curve shape can give rise to the observed data. When it comes to determining an accurate slope or detailed shape for an individual galaxy, beam smearing correction are frequently ambiguous.

\section{Deriving hybrid rotation curves}

In many cases the HI data extend further out than the $\mathrm{H} \alpha$ data. Due to the flattening of the Hi curve towards larger radii, resolution effects become less important, and to get a more extended rotation curve it therefore makes sense to combine the $\mathrm{HI}$ and $\mathrm{H} \alpha$ data. We have defined hybrid $\mathrm{HI} / \mathrm{H} \alpha$ curves, always giving preference to $\mathrm{H} \alpha$ data where available. In practice this meant that we simply adopt the $\mathrm{H} \alpha$ rotation velocities interior to a radius $r_{\mathrm{H} \alpha}$, usually
Table 3. Combining Hi $/ \mathrm{H} \alpha$.

\begin{tabular}{lrlr}
\hline Name & $r_{\mathrm{H} \alpha}\left({ }^{\prime \prime}\right)$ & Name & $r_{\mathrm{H} \alpha}\left({ }^{\prime \prime}\right)$ \\
\hline F563-1 & 60 & N1560 & 200 \\
U628 & $-{ }^{a}$ & IC2233 & $-^{b}$ \\
U711 & $-{ }^{a}$ & N2366 & 200 \\
U731 & 40 & N3274 & 70 \\
U1230 & 90 & DDO64 & 80 \\
U1281 & 115 & DDO52 & 80 \\
U3137 & 88 & DDO47 & 100 \\
U3371 & 104 & DDO185 & $-{ }^{b}$ \\
U4173 & 10 & DDO189 & 60 \\
U4325 & $-{ }^{b}$ & N5023 & 180 \\
U5005 & 65 & N4455 & 100 \\
U5750 & 39 & N4395 & 250 \\
N100 & $-{ }^{a}$ & U10310 & 70 \\
\hline
\end{tabular}

Notes: ${ }^{a}$ No Hi curve; ${ }^{b}$ Hi not used.

corresponding to the outermost point of the $\mathrm{H} \alpha$ rotation curve, and the Hi velocities outside that radius. Table 3 lists these radii, along with supplementary notes.

\subsection{Asymmetric drift}

In order to combine the $\mathrm{HI}$ and $\mathrm{H} \alpha$ rotation curves one should in principle correct for the pressure gradients in the gas. This correction for asymmetric drift is given by

$v_{\mathrm{c}}^{2}=v_{\phi}^{2}-\sigma^{2}\left(\frac{\partial \ln \rho}{\partial \ln R}+\frac{\partial \ln \sigma^{2}}{\partial \ln R}\right)$

where $v_{\mathrm{c}}$ is the true rotation velocity, $v_{\phi}$ the observed gas rotation velocity, $\sigma$ the velocity dispersion in the gas, and $\rho$ the volume density. The velocity dispersion in late-type galaxies is found to lie between 7 and $10 \mathrm{~km} \mathrm{~s}^{-1}$ and is to first order constant with radius. Assuming a constant scale height we then find that the correction for asymmetric drift is typically $\sim 3 \mathrm{~km} \mathrm{~s}^{-1}$ or less. Given other uncertainties due to non-circular motions, inclination, etc. the asymmetric drift corrections are negligible and we have therefore not applied this correction.

\subsection{Deriving smooth rotation curves}

Some of the main assumptions that are made when deriving mass models from rotation curves is that there is symmetry, that all mass is on circular orbits and there is continuity with radius. The raw data still show some scatter and non-circular motions, which can occasionally result in virtual or ambiguous rotation velocities. In order to estimate the (smooth) radial run of the potential we want to represent the data by a curve whose velocity and velocity-derivative change smoothly, retaining smallscale details. As we are combining $\mathrm{HI}$ and $\mathrm{H} \alpha$ points we also want the density of the different data points to be 
approximately equal, in order to prevent the densely sampled $\mathrm{H} \alpha$ curves from dominating the fits. We have used a local regression method to derive the smooth curve (Loader 1999). This method fits polynomial pieces locally to subsets of data but does not impose any global functional description (see dBMR for a more extensive description). As this process inevitably introduces a small degree of correlation between adjacent data points, the smooth $(\mathrm{H} \alpha)$ curves were resampled every $6^{\prime \prime}$.

The final errorbar in these resampled data points consists of two components: (i) the average measurement uncertainty, derived from the average weighted measurement error in each $6^{\prime \prime}$ interval; (ii) an error estimate due to largescale asymmetries and non-circular motions. We used the difference between the weighted mean velocity and the smooth curve in each interval as an estimate. These two uncertainties were added quadratically giving a conservative estimate for the uncertainty.

A general problem with $\mathrm{H} \alpha$ rotation curves is that the formal errorbars of well-sampled, high $S / N$ data points become unrealistically small (sometimes less than $1 \mathrm{~km} \mathrm{~s}^{-1}$ ). These errorbars no longer have any physical significance but simply indicate that the Gaussian fit to the profile was well-determined. A similar problem also exists with HI curves where the tilted-ring fitting programs produce error bars that give the uncertainty in the fit and not the actual uncertainty due to profile width etc. Realistically, observational and physical uncertainties make it difficult to determine a meaningful rotation velocity with an accuracy more than a few $\mathrm{km} \mathrm{s}^{-1}$. As the final step in our derivation of the rotation curves we have imposed a minimum error of $4 \mathrm{~km} \mathrm{~s}^{-1}$ (prior to inclination correction) on each data point.

We compare the resampled smooth curves with the raw data in Fig. 7. To avoid cluttering the plot we represent the smooth curves as a line. The full curves, complete with data points, are also shown in Fig. 8. It is evident that the method does not introduce any systematic effects that affect the shape of the curve. For an extensive discussion see dBMR. The curves shown in Fig. 7 will be used as the basis for our mass modelling.

\section{Mass models}

In order to find the signature of the dark matter halo one needs to decompose the observed rotation curve in a number of separate dynamical components, described below.

\subsection{Stellar and gas components}

For the stellar disk the $R$-band photometry presented in de Blok et al. (1995) and Swaters (1999) (see also Swaters et al. 2002a) was used. For NGC 1560 we used the $B$ band photometry presented in Broeils (1992). For DDO47 and DDO64 we used the radial surface brightness profiles from Stil (1999). These profiles are presented as relative to the sky-level without absolute calibration and we therefore used the surface photometry presented in Hopp \& SchulteLadbeck (1991) and Makarova (1999) to put the profiles on an absolute $R$-band surface brightness scale. (Since the Stil photometry is in the $I$-band, this assumes that there are no strong $R-I$ gradients.) See Table 1 for more details.

The rotation curve of the disk was computed using the ROTMOD task in GIPSY. The disk was assumed to have a vertical $\operatorname{sech}^{2}$ distribution with a scale height $z_{0}=h / 6$ (van der Kruit \& Searle 1981). The rotation curves of the stellar component were resampled at the same radii as the smooth curves. We assume $M / L_{\star}$ constant with radius, which is a reasonable assumption for the range of variation expected from plausible stellar populations (de Jong 1996).

For the gas component we used the Hi surface densities from the references given in Table 1 . They were scaled by a factor of 1.4 to take into account the contribution of helium and metals. Their rotation curve was derived assuming the gas is distributed in a thin disk. For a few nearby galaxies we had to slightly smooth the Hi radial profile, mainly because small-scale structure at small radii cause unrealistically large fluctuations in the gas rotation curve and hence the potential. At large radii this smallscale structure is averaged out so the HI profile there is a good description of the global Hi structure.

\subsection{Dark halo}

After subtraction of the above components from the observed rotation curve, any residuals are usually taken to indicate the presence of a dark matter halo. The halos are usually parametrised by fitting a model to these residuals. The quality of the fit tells us something about the applicability of any particular model. Here we restrict ourselves to two well-known models: the pseudo-isothermal halo, used in most of the classic rotation curve studies, and the CDM NFW halo.

\subsubsection{Pseudo-isothermal halo}

We assume a spherical pseudo-isothermal halo with a density profile

$\rho_{\mathrm{ISO}}(R)=\rho_{0}\left[1+\left(\frac{R}{R_{\mathrm{C}}}\right)^{2}\right]^{-1}$

where $\rho_{0}$ is the central density of the halo, and $R_{\mathrm{C}}$ the core radius of the halo. This density profile results in the rotation curve

$$
V_{\text {halo }}(R)=\sqrt{4 \pi G \rho_{0} R_{\mathrm{C}}^{2}\left[1-\frac{R_{\mathrm{C}}}{R} \arctan \left(\frac{R}{R_{\mathrm{C}}}\right)\right]}
$$



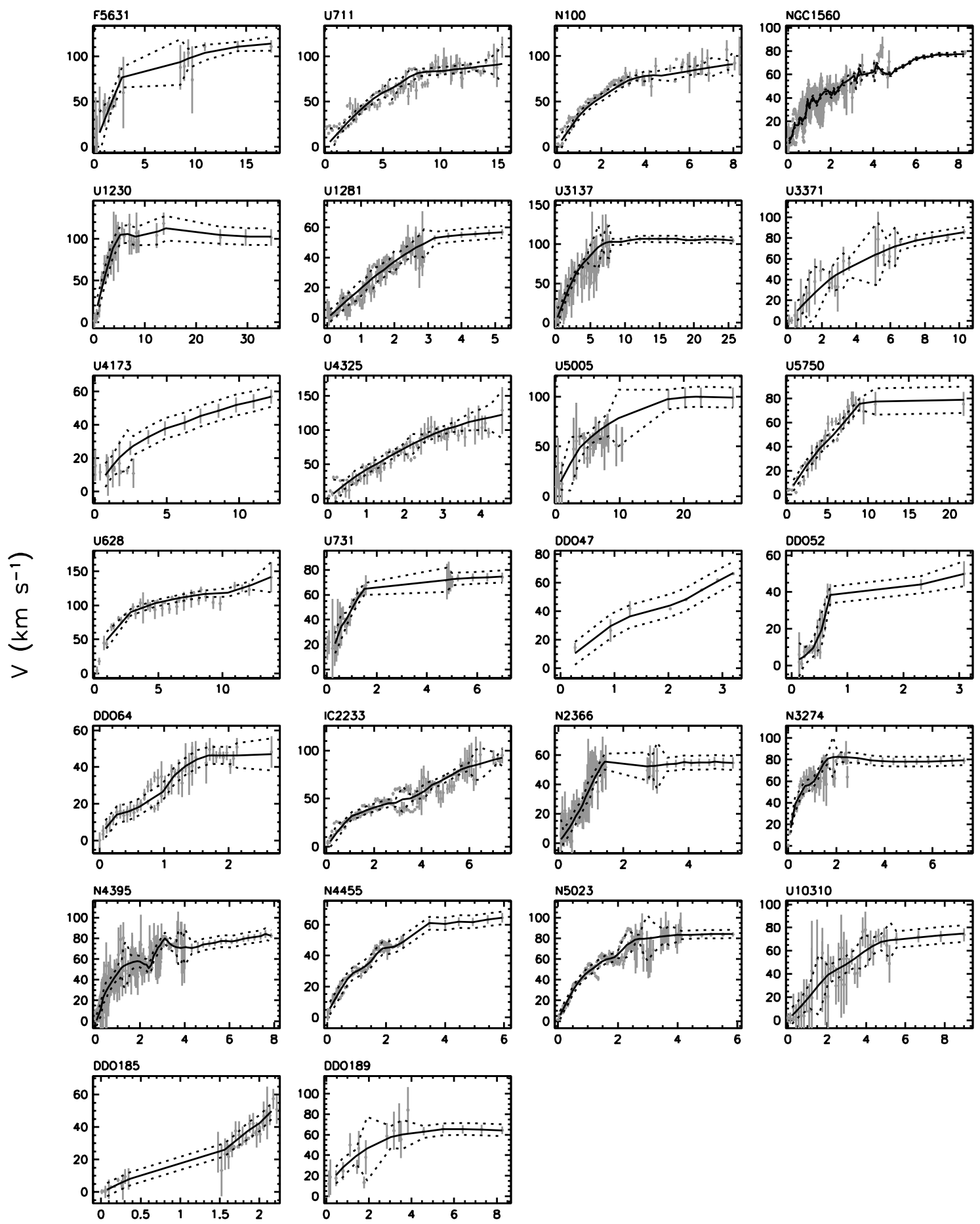

$r(k p c)$

Fig. 7. Final hybrid smooth rotation curves (full and dashed line) overlaid on original raw hybrid H $\alpha \mathrm{HI}$ data (grey symbols). The smooth curves are a good representation of the raw data.

The asymptotic velocity of the halo, $V_{\infty}$, is given by

$$
V_{\infty}=\sqrt{4 \pi G \rho_{0} R_{\mathrm{C}}^{2}}
$$

To characterise this halo only two out of the three parameters $\left(\rho_{0}, R_{\mathrm{C}}, V_{\infty}\right)$ are needed, as Eq. (3) determines the value of the third parameter.

\subsubsection{NFW halo}

The NFW mass density distribution (Navarro et al. 1996, 1997) takes the form

$\rho_{\mathrm{NFW}}(R)=\frac{\rho_{i}}{\left(R / R_{\mathrm{s}}\right)\left(1+R / R_{\mathrm{s}}\right)^{2}}$ 
where $R_{\mathrm{S}}$ is the characteristic radius of the halo and $\rho_{i}$ is related to the density of the universe at the time of collapse. These parameters are not independent and are set by the cosmology. They are usually expressed in a slightly different form by the concentration parameter $c=R_{200} / R_{\mathrm{s}}$, and the rotation velocity $V_{200}$ at radius $R_{200}$. The latter is the radius where the density contrast exceeds 200, roughly the virial radius (Navarro et al. 1996). The rotation curve of the NFW halo then is given by

$V(R)=V_{200}\left[\frac{\ln (1+c x)-c x /(1+c x)}{x[\ln (1+c)-c /(1+c)]}\right]^{1 / 2}$,

where $x=R / R_{200}$.

\subsection{Stellar mass-to-light ratios}

One of largest uncertainties in any mass model is the precise value of $M / L_{\star}$. Though broad trends in $M / L_{\star}$ have been measured and modelled (Bottema 1997; Bell \& de Jong 2000), the precise value from galaxy to galaxy is unknown, and depends on extinction, star formation history, initial mass function, etc. Because of this problem we present disk-halo decompositions using four different assumptions for $(M / L)_{\star}$ for those galaxies with photometry available. For the galaxies without photometry only the first (minimum-disk) model is presented.

1. Minimum disk. This ignores all visible components and assumes that the observed rotation curve is entirely due to dark matter. This gives a strong limit on the amount of dark matter in a galaxy. This is what is usually called "minimum disk" in the CDM analyses;

2. minimum disk + gas. This is the classical definition of minimum disk mostly used in observational rotation curve papers (and usually called "minimum disk" there). The contribution of the gas is taken into account, but $M / L_{\star}$ is assumed to be zero;

3. constant $M / L_{\star}$. Here $M / L_{\star}$ is fixed to a constant and representative value for all galaxies. In this case we have chosen $M / L_{\star}(R)=1.4$ which is at the "blue" or light-weight end of the range of plausible values derived (under reasonable assumptions for IMF and star formation history) from population synthesis models using the observed colours as constraints. See dBMR for a further motivation of this value. By using this "lightweight" value we give the halo models maximum opportunity to fit the data;

4. maximum disk. Here the rotation curve of the stellar component is scaled to the maximum value allowed by the smooth curve, without exceeding it (van Albada \& Sancisi 1986). In practice the stellar curve is scaled until the inner points match those of the smooth curve, but avoiding a hollow halo.

Each of the rotation curves was fitted using the GIPSY task ROTMAS. The program determines the best-fitting combination of $R_{\mathrm{C}}$ and $V_{\infty}$ (for the pseudo-isothermal halo) or $c$ and $V_{200}$ (for the NFW halo), using a least squares fitting routine.

\section{Modelling results}

The results of the modelling are presented in Fig. 8 and Tables 4 and 5 . Figure 8 displays the models for the four $M / L_{\star}$ assumptions, or, if no photometry was available, only the minimum disk model. The NFW and pseudoisothermal ("ISO") halo fits are shown side by side. The values of the halo parameters are given in Table 4 for the NFW model and Table 5 for the pseudo-isothermal halo model. Both figure and tables also list the reduced $\chi^{2}$ goodness-of-fit values. We have also done a simple $\chi^{2}$ test to compute the chance $p$ that the data and the models could originate from the same distribution. We regard $p>0.95$ as a good fit, indicating that the data and the model match. Values of $p<0.05$ generally indicate that the wrong model is used to describe the data. Derived values for $p$ are shown in Fig. 8 .

For the NFW fits the fitting routine often preferred very small or negative $c$ values. These are obviously unphysical, and we have restricted the parameter space for $c$ to values $\geq 0.1$. When the fit preferred a value less than 0.1 we set $c$ simply equal to 0.1 , and found the best fitting value for $V_{200}$. This is indicated in Table 4 by giving the values in italics.

\subsection{Comments on the mass modelling of individual galaxies}

F563-1: The pseudo-isothermal halo fits are comparable in quality to the NFW fits. We can compare our results with the independent fits by dBMR. For the minimum disk pseudo-isothermal halo we find $R_{\mathrm{C}}=2.0 \pm 0.2$ and $\rho_{0}=70.4 \pm 13.1$. dBMR find $R_{\mathrm{C}}=1.7 \pm 0.2$ and $\rho_{0}=91.9 \pm 21.6$. These values thus agree within their $1 \sigma$ errors. For the NFW models our values of $c=7.8 \pm 1.4$ and $V_{200}=106.8 \pm 10.3$ compare well with the dBMR values of $c=10.7 \pm 1.2$ and $V_{200}=93.1 \pm 4.3$. The $c$ values agree at the $1.1 \sigma$ level, whereas the $V_{200}$ values agree within the $1 \sigma$ errorbars. Two completely independent analyses of different observations on different telescopes are thus able to reproduce the same parameters, showing that systematic effects due to telescope pointing, smoothing etc. are small.

U628: The pseudo-isothermal and NFW fits are comparable. One should note that for this galaxy no Hi data are available, and that the "minimum disk" and "minimum disk+gas" fits are identical. Also no gas rotation curve is included in the "constant $M / L_{\star}$ " and "maximum disk" cases. The gas rotation curve does however usually have only a small impact on the fit parameters, and the values presented here will be close to the true ones.

U731: The pseudo-isothermal models and NFW fits are comparable except that the latter overestimate the rotation velocity in the inner parts. Both models have trouble fitting the sharp kink at $r \sim 0.8 \mathrm{kpc}$. This kink is artificial and caused by a lack of data between 0.8 and $4.2 \mathrm{kpc}$ due to the presence of a strong night-sky line at these velocities. 

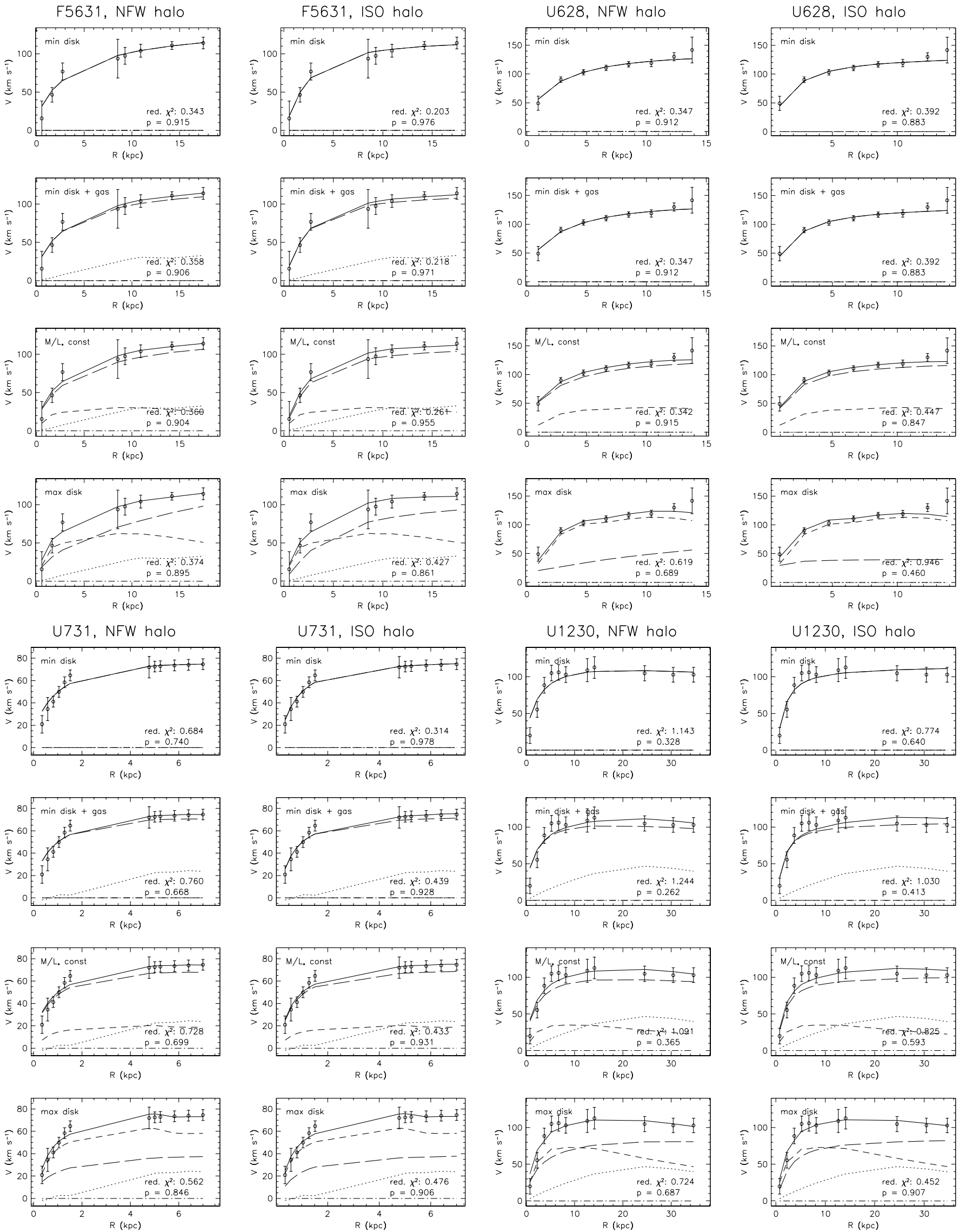

Fig. 8. Mass models for F563-1, U628, U731 and U1230. First and third column show fits using the NFW halo model; second and fourth column show models using the pseudo-isothermal halo model. Reduced $\chi^{2}$ and $p$-values are given in the bottom-right of each panel. 
U3371, NFW halo
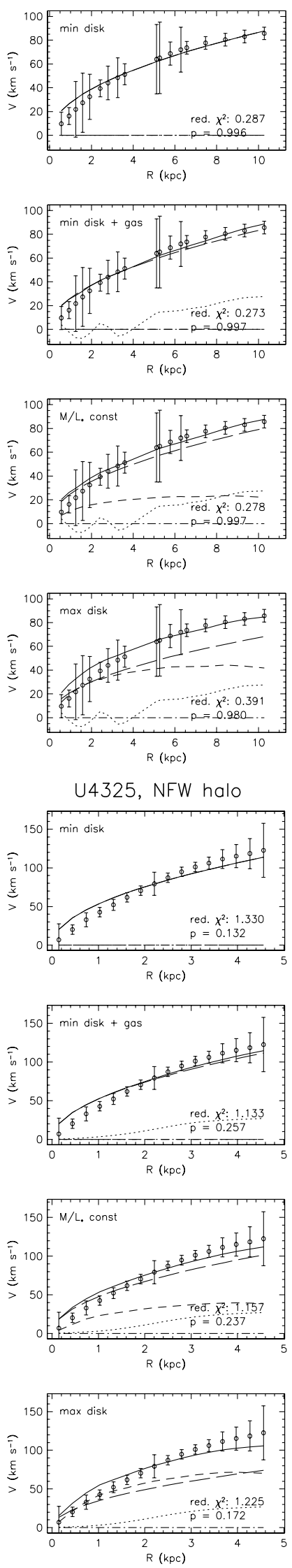
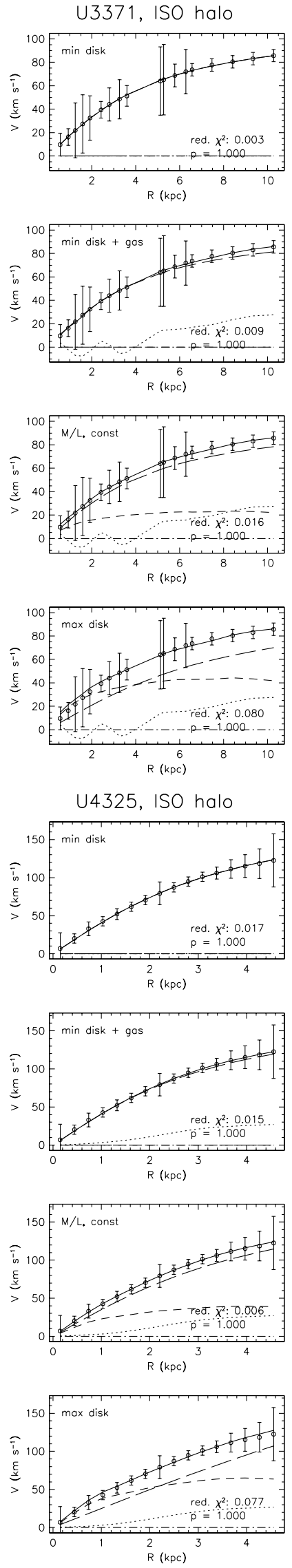
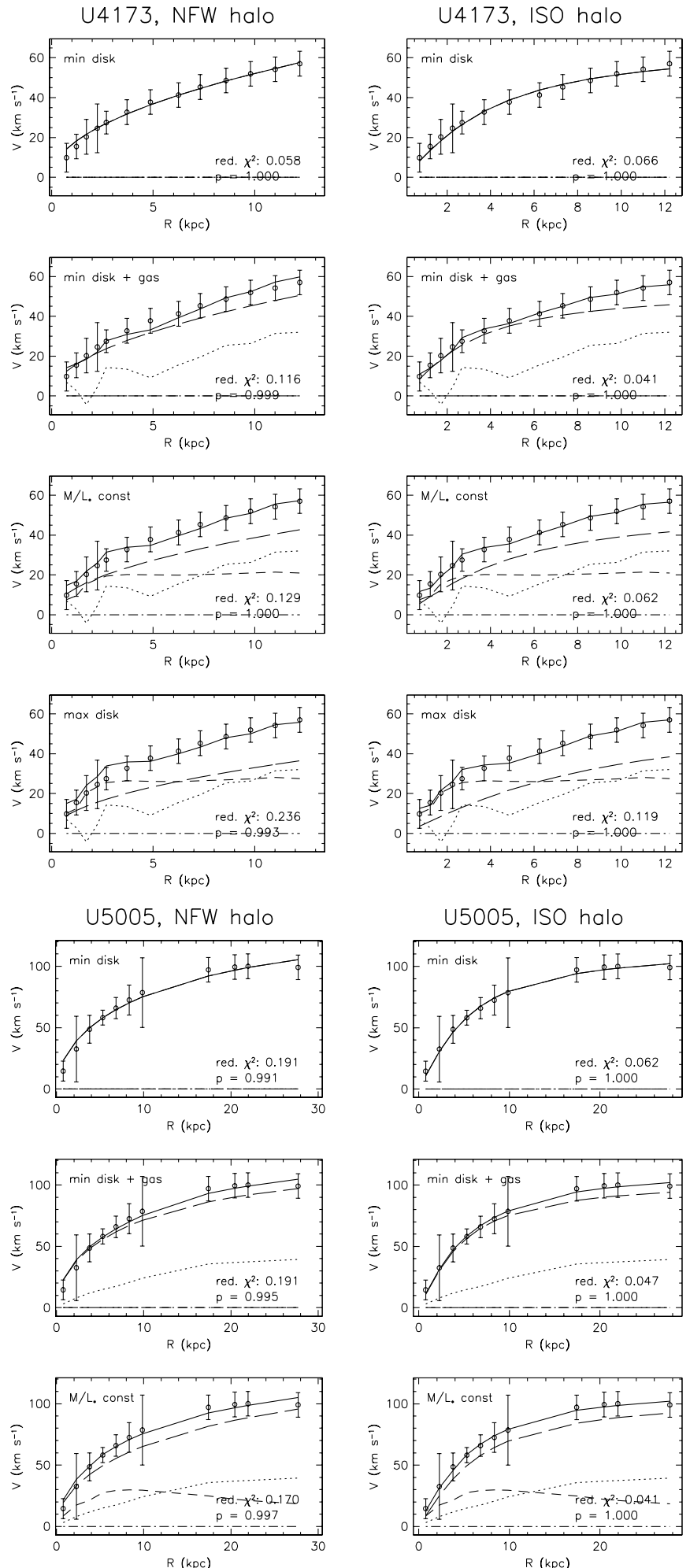

U5005, ISO halo
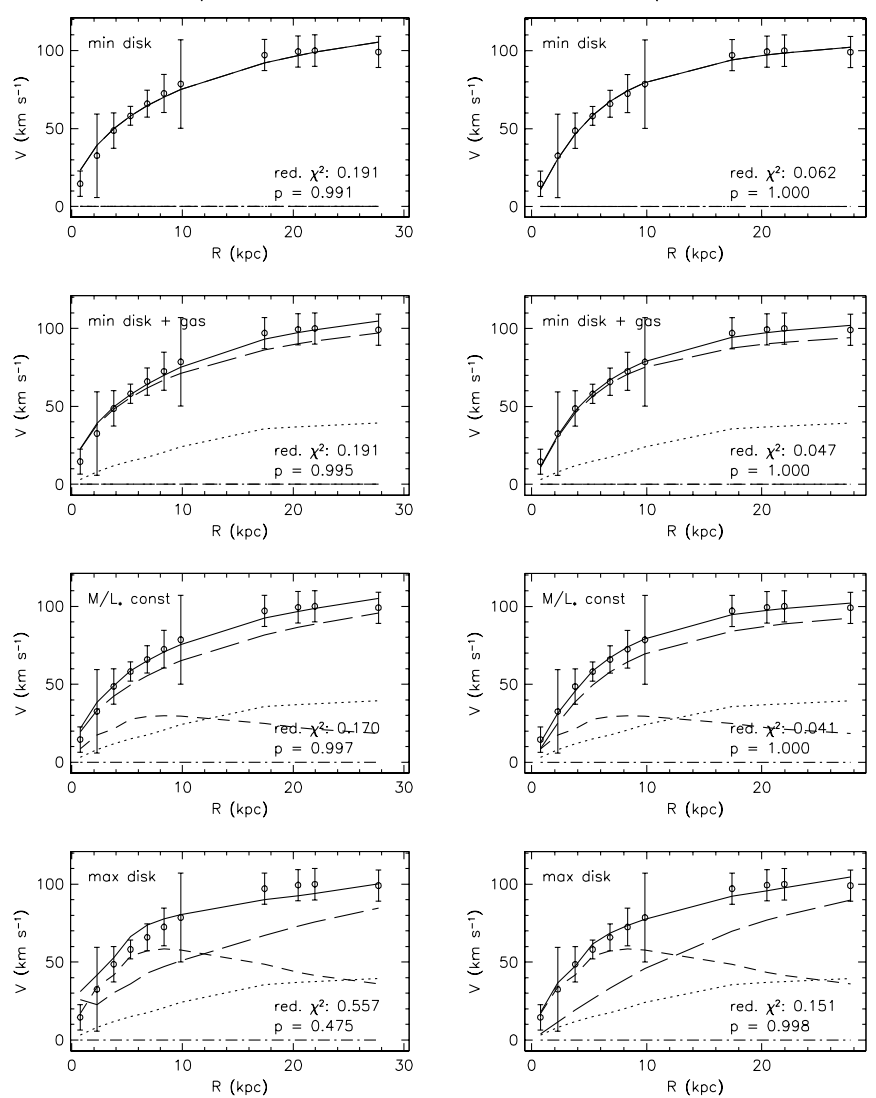

Fig. 8. continued. Mass models for U3371, U4173, U4325 and U5005. 
U5750, NFW halo
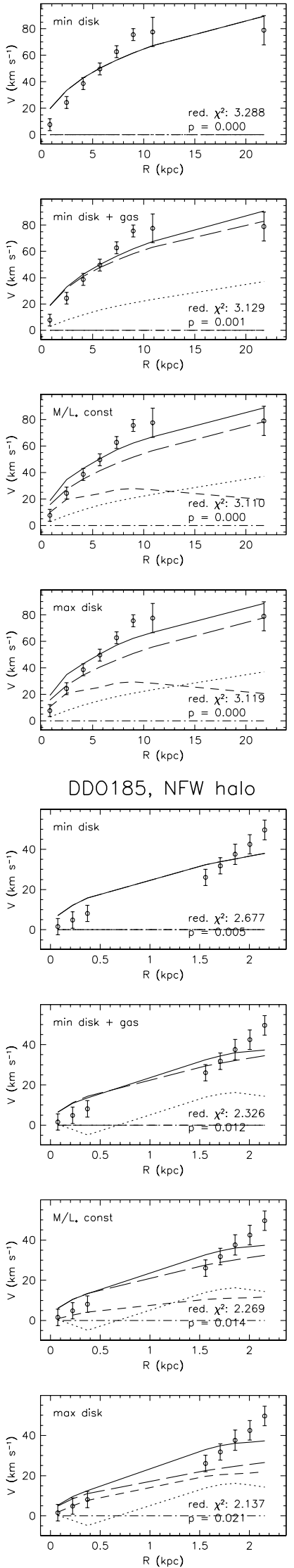

U5750, ISO halo
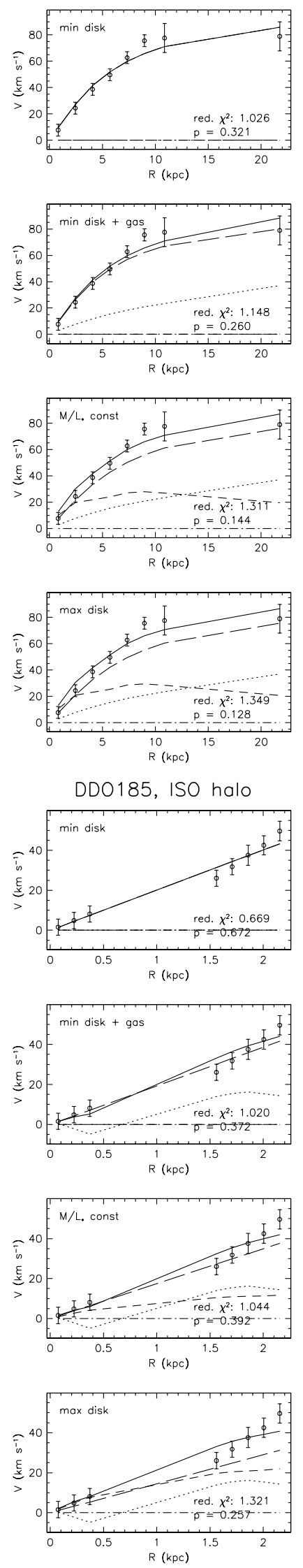
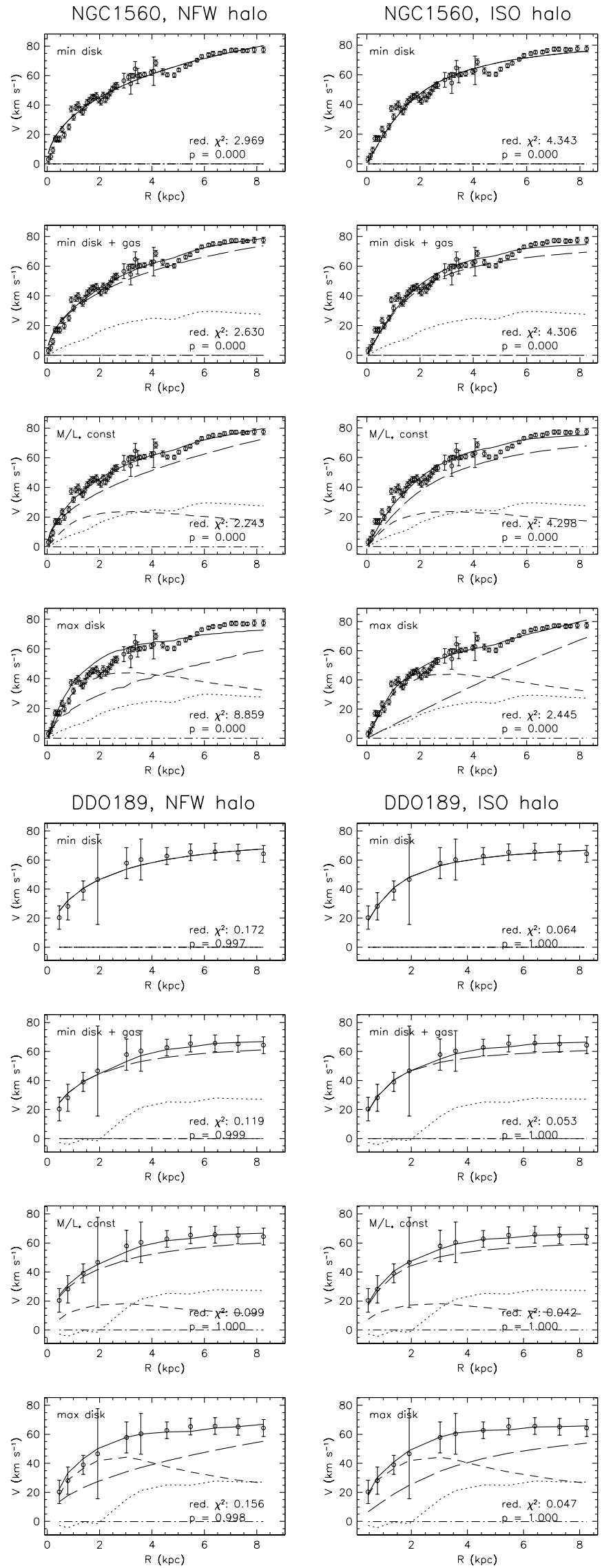

Fig. 8. continued. Mass models for U5750, N1560, DDO185 and DDO189. 

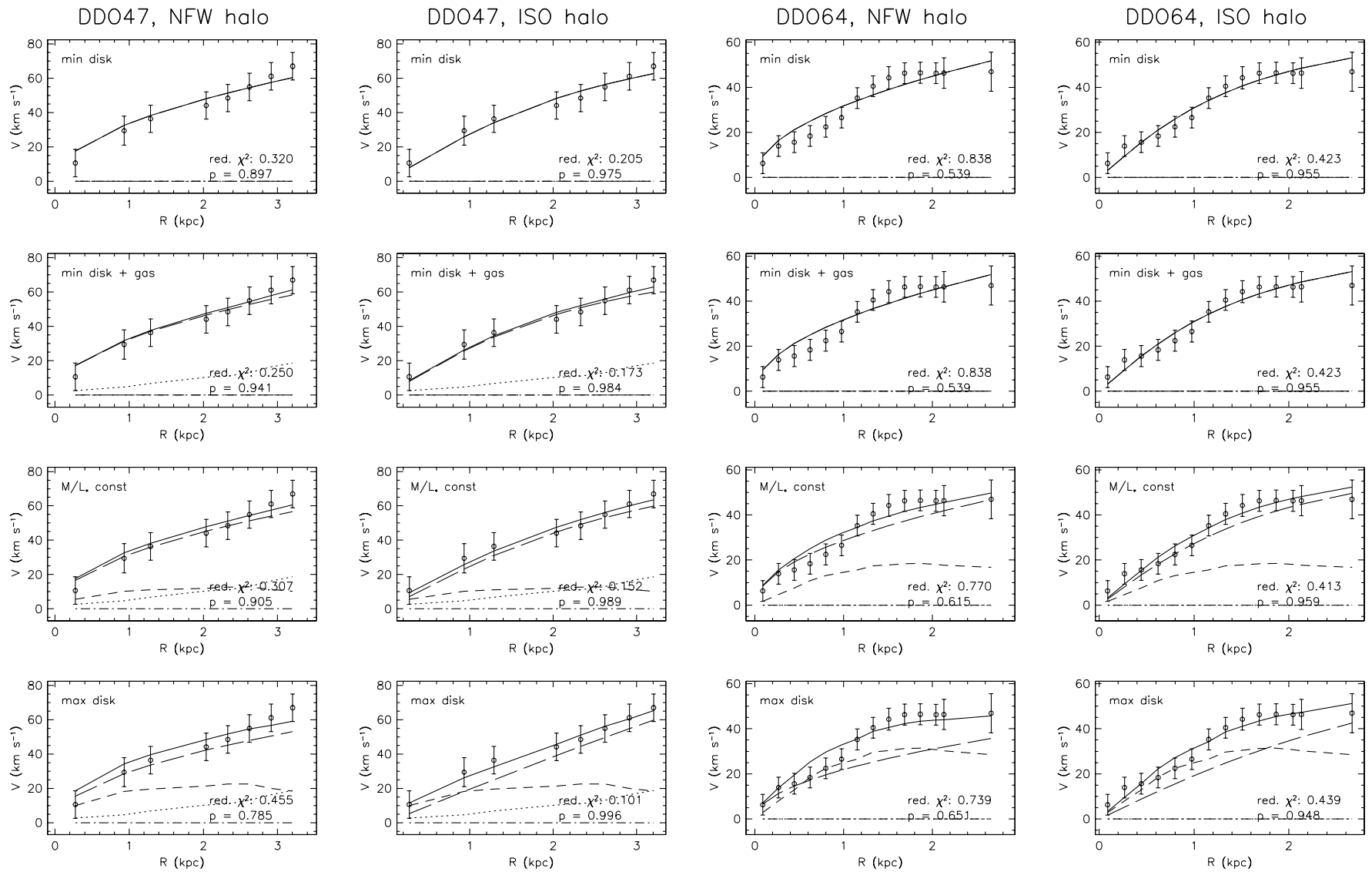

N2366, NFW halo

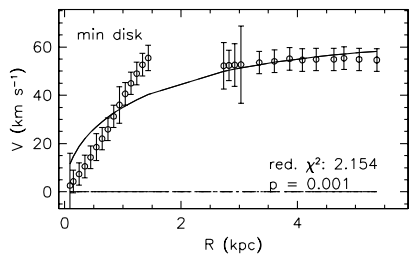

N2366, ISO halo

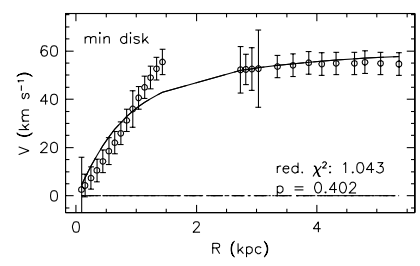

N3274, NFW halo
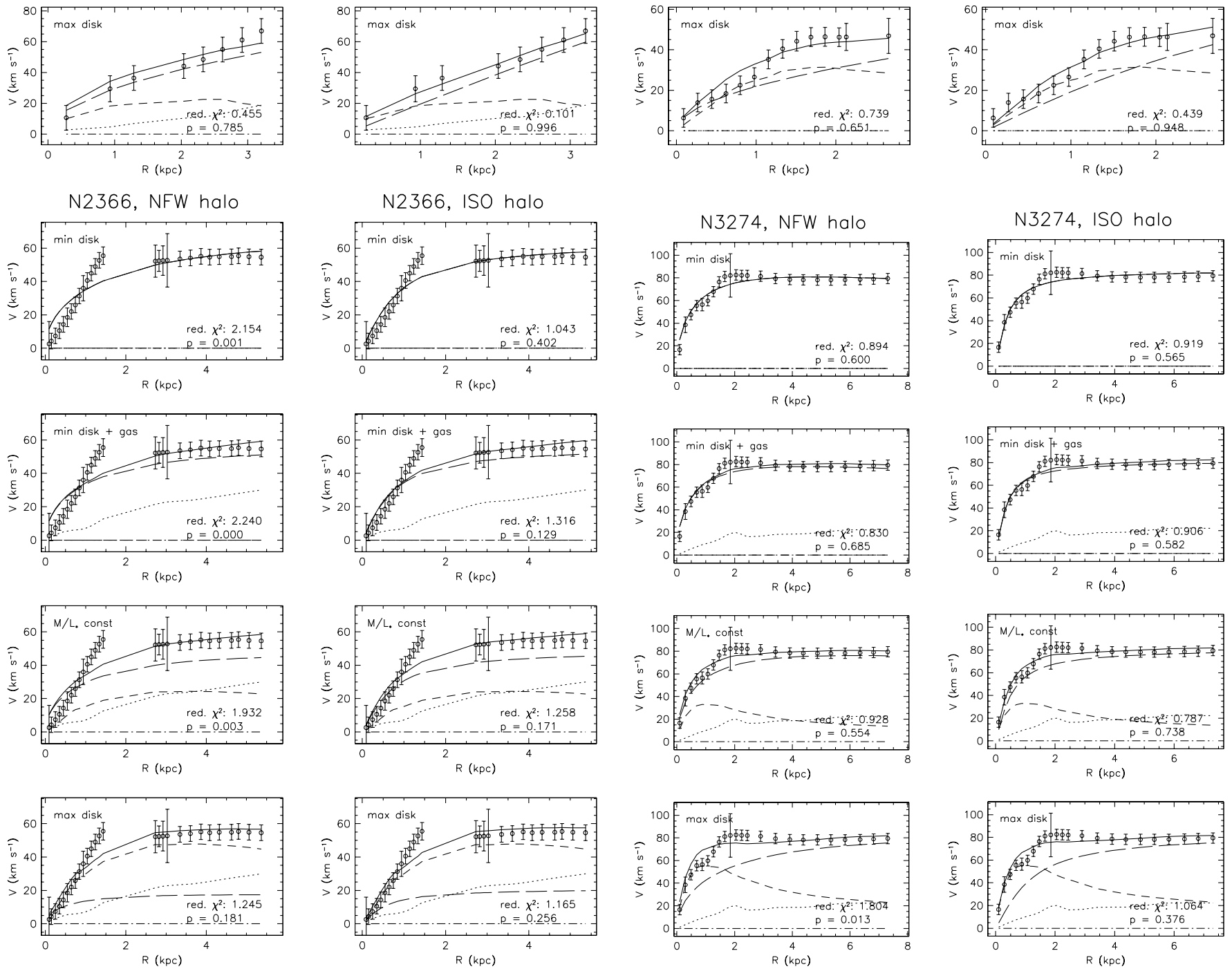

Fig. 8. continued. Mass models for DDO47, DD064, N2366 and N3274. 

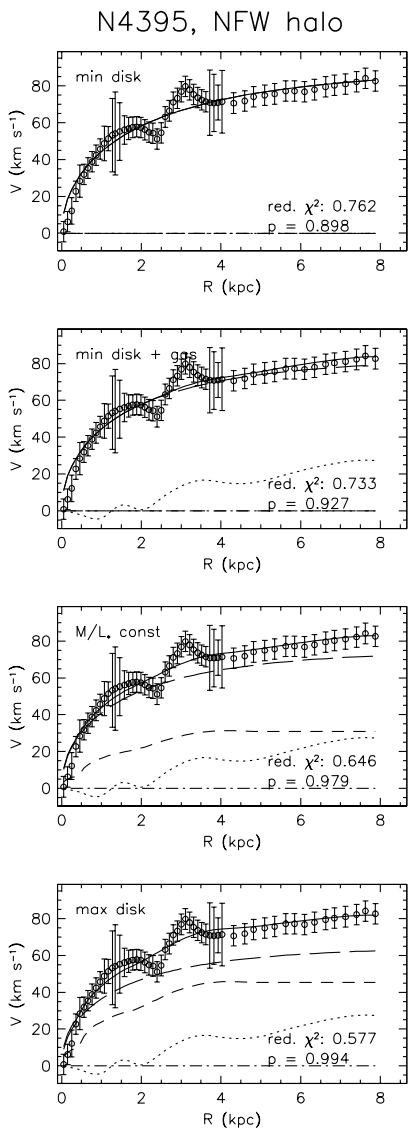
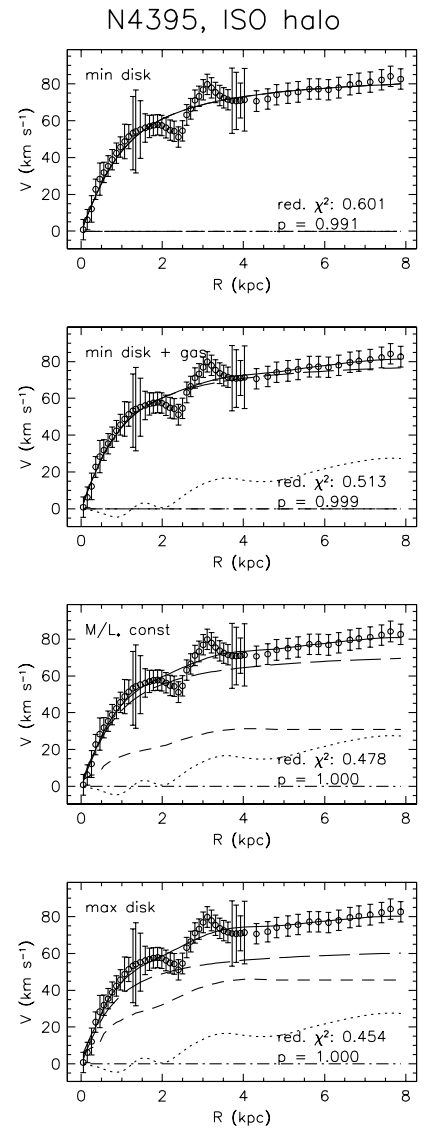

Fig. 8. continued. Mass models for N4395 and N4455.

U1230: For both models maximum disk is the best fit. This comes at the cost, however, of a high $M / L_{\star}(R)=6.1$ which is hard to explain in term of reasonable population synthesis $M / L_{\star}$ values, which usually find values between $\sim 0.5$ and $\sim 2$ for LSB galaxies (van den Hoek et al. 2000).

U4173: This rotation curve is virtually identical to the Hi curve as the $\mathrm{H} \alpha$ data do not add much extra constraint. Both models fit equally well. The Hi surface density profile given in Swaters (1999) gives rise to large fluctuations in the inner gas rotation curve and was therefore smoothed slightly as explained above.

U4325: Good fits for the pseudo-isothermal models, bad ones for the NFW models.

U5005: Both sets of fits are comparable. The data are however not of high enough quality to constrain either model.

U5750: This galaxy can again be compared with independent observations by dBMR. We first compare the fitting parameters. For the isothermal model we find $R_{\mathrm{C}}=$ $5.0 \pm 0.9$ and $\rho_{0}=7.9 \pm 1.6 . \mathrm{dBMR}$ find $R_{\mathrm{C}}=4.3 \pm 0.4$ and $\rho_{0}=10.6 \pm 1.0$ (again minimum disk). These agree within the $1 \sigma$ level. For the NFW model we find $c=1.9 \pm 2.1$ and $V_{200}=145.7 \pm 122.9$, compared to their $c=2.6 \pm 1.5$ and $V_{200}=123.1 \pm 58.8$, again in good agreement. The large uncertainties in the NFW parameters indicate that the rotation curve of U5750 cannot be adequately described by that model. This is mainly caused by the very linear rise of the inner rotation curve. For the NFW model the only
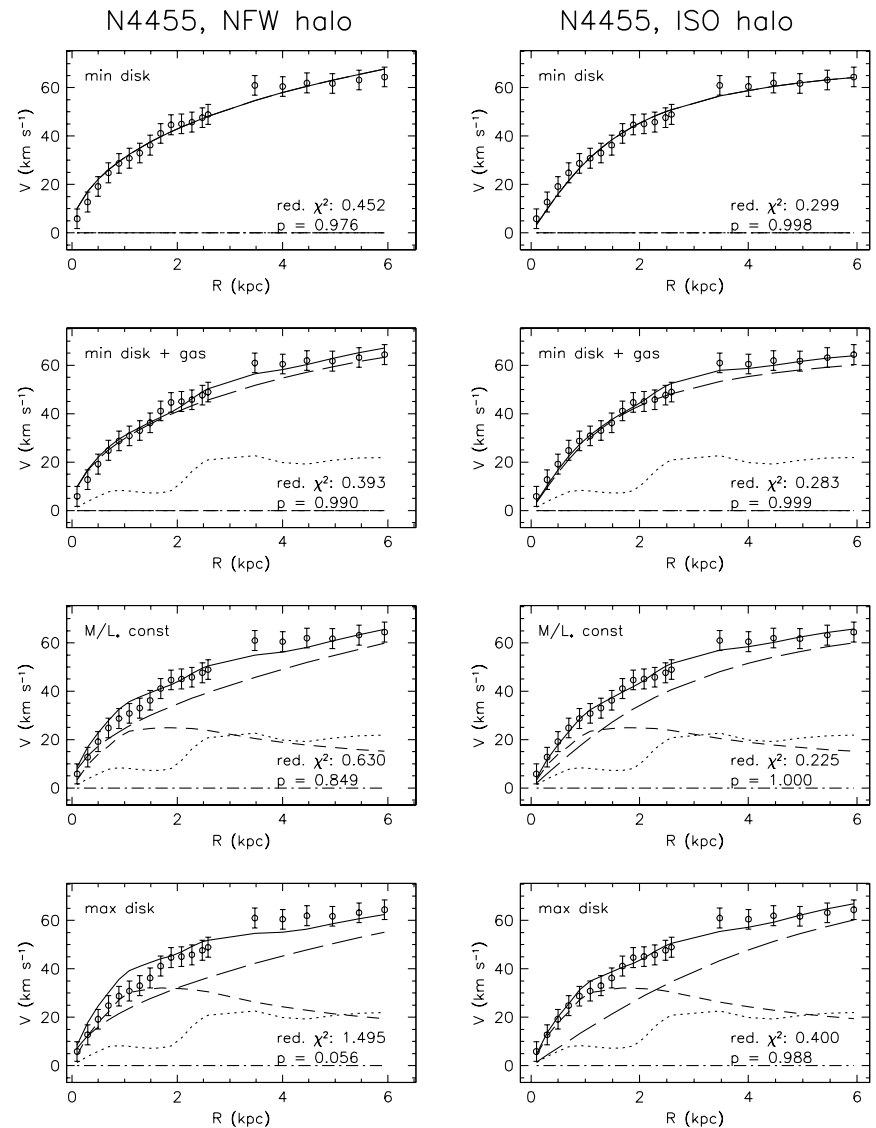

constraint is imposed by the two Hi points at the flat part of the curve. Without these the fit would diverge to $c=0$ and $V_{200}=\infty$. Clearly, higher-resolution Hi observations of the outer parts are needed further fill in the flat part of the curve and bring down the uncertainties of the fit parameters. This would however not change the conclusion that this galaxy is very hard to fit with the NFW model. Again we see that independent observations are capable of reproducing the same set of parameters at the $1 \sigma$ level.

N1560: Due to the large number of data points and the small scale structure present in the rotation curve, the $\chi^{2}$ values are high. The pseudo-isothermal and NFW models seem to perform equally well, though there is still a slight tendency of the NFW models to overestimate the inner parts. Whereas the problem with rotation curves is usually that there is not enough resolution, NGC 1560 illustrates the opposite: the non-circular motions in the $\mathrm{H} \alpha$ data are large. For the pseudo-isothermal model maximum disk fits best, but again with a value $M / L_{\star}(B)=4.9$ which is much higher than one would find on the basis of reasonable star formation histories and stellar initial mass functions.

DD0185: This is a very linear curve that is reasonably fitted by pseudo-isothermal models, but poorly by NFW models. The curve is however only of average quality, and better observations are needed to constrain the models further. 
U711, NFW halo

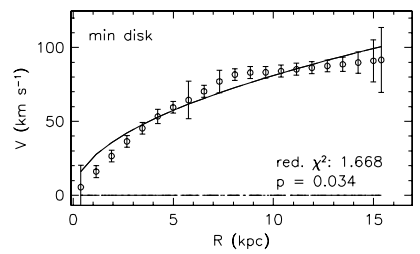

U3137, NFW halo

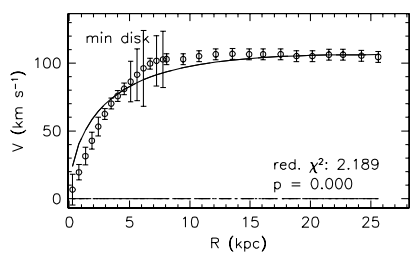

DD052, NFW halo

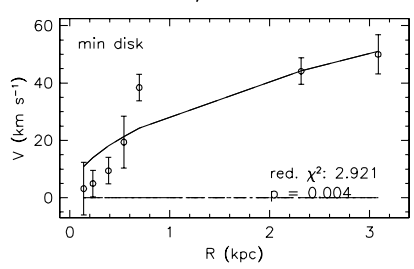

N5023, NFW halo

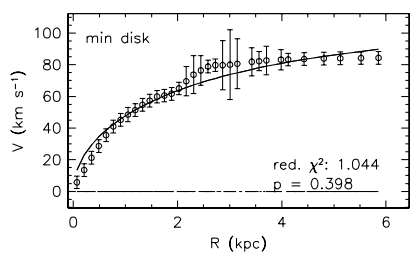

U711, ISO halo

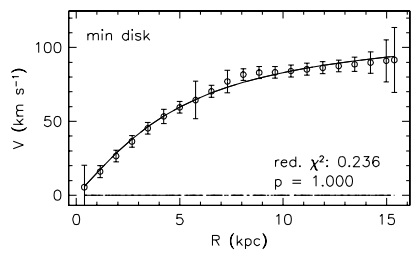

U3137, ISO halo

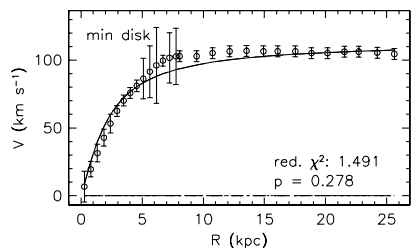

DD052, ISO halo

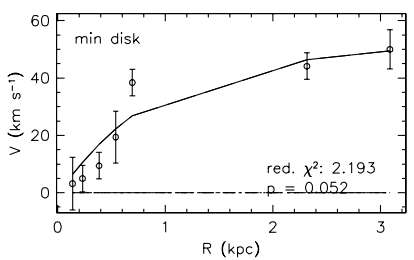

N5023, ISO halo

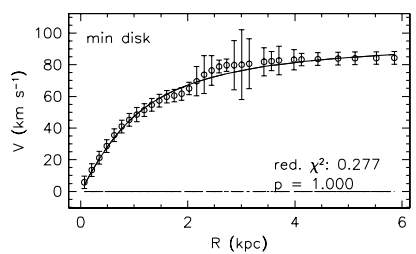

U1281, NFW halo

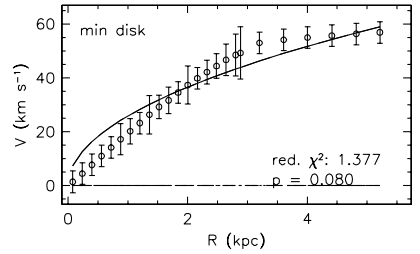

N100, NFW halo

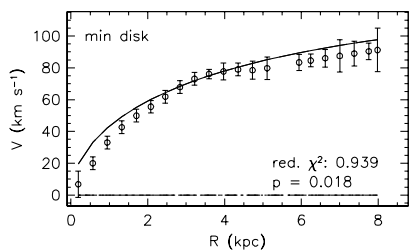

IC2233, NFW halo

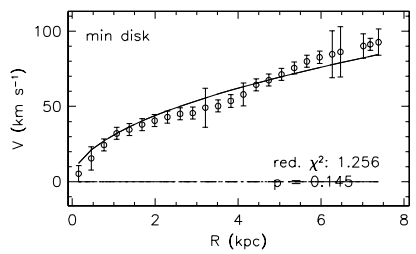

U10310, NFW halo

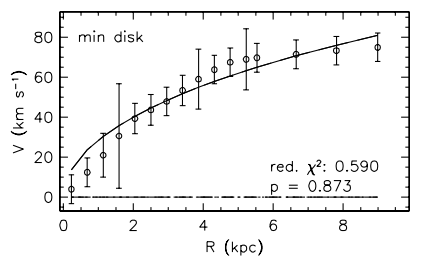

U1281, ISO halo

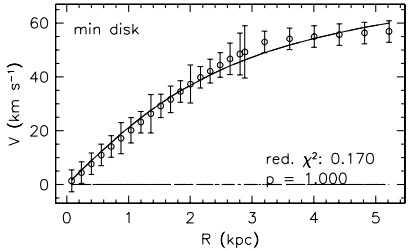

N100, ISO halo

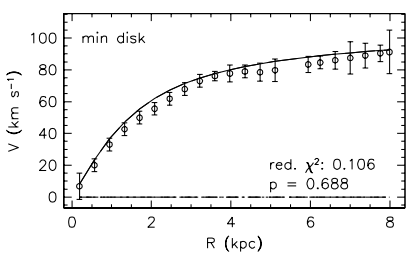

IC2233, ISO halo

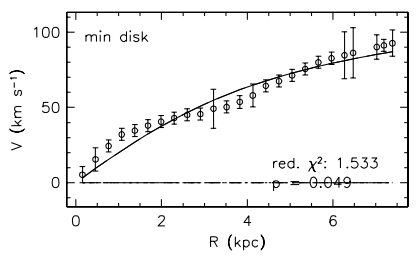

U10310, ISO halo

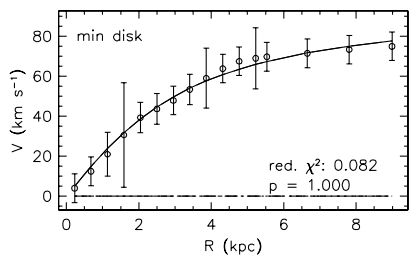

Fig. 8. continued. Mass models for galaxies with only minimum disk fits.

DDO189: This curve is equally well fitted by pseudoisothermal and NFW models.

DDO64: This curve shows a bump in the inner parts, that is very well fitted by the stellar disk in the maximum disk model, perhaps indicating that the inner part of this galaxy is dominated by stars. Similar bumps have been found in other highly resolved observations of dwarf galaxies (e.g. Blais-Ouellette et al. 2001). The pseudoisothermal model is a better fit.

N2366: This curve is a challenge for both ISO and NFW models. The very linear rise in the inner parts rapidly changes in a flat part at larger radii. It is hard to reproduce such a sharp change in slope. It is possible that the Hi curve on which the outer points are based underestimates the rotation velocity as a comparison with the $\mathrm{HI}$ position-velocity diagram perhaps suggests. Another possible explanation would be that non-circular motions due to the bar-like structure in the centre affect the curve. In their study of NGC 2366 Hunter et al. (2001) find some weak evidence for this from the HI velocity field, but they also note that the rotation curve does not seem to be affected. Clearly there is room for additional high-resolution velocity measurements.
N3274, N4395, N4455: The NFW models fit as well as the ISO models. Parts of the rotation curve of N4395 are dominated by effects of star formation.

U1281, U3137, U711, N100, DDO52, N5023, IC2233 and U10310: For these eight galaxies only minimum disk models are presented. For U711, N100, DDO52 and U10310 this is because no surface photometry is available. U1281, U3137, N100, IC2233, and N5023 are edge-on galaxies and deriving their face-on surface brightness profiles depends on various assumptions about the properties of their disk which would introduce additional uncertainties in the models.

\subsection{Goodness of fit}

The $\chi^{2}$ values of the pseudo-isothermal models are generally smaller than those of the NFW models. This is illustrated in Fig. 9 where we plot both sets of $\chi^{2}$ values against each other for the four different $M / L_{\star}$ scenarios. In all cases the NFW models are either comparable or worse than the isothermal models. We emphasise this point again in Table 6 . Here we list the number of galaxies with good $(p>0.95)$ and bad $(p>0.05)$ fits for the two models under the four $M / L_{\star}$ assumptions. 
Table 4. Parameters NFW models.

\begin{tabular}{|c|c|c|c|c|c|c|c|c|c|c|c|c|c|}
\hline Name & $c$ & $\Delta c$ & $V_{200}$ & $\Delta V$ & $\chi^{2}$ & $p$ & $c$ & $\Delta c$ & $V_{200}$ & $\Delta V$ & $\chi^{2}$ & $p$ & $\Upsilon_{*}^{\max }$ \\
\hline & \multicolumn{6}{|c|}{ Minimum disk } & \multicolumn{6}{|c|}{ Minimum disk + gas } & \\
\hline F5631 & 7.8 & 1.4 & 106.8 & 10.3 & 0.343 & 0.915 & 8.1 & 1.5 & 99.7 & 9.4 & 0.358 & 0.906 & \\
\hline U5750 & 1.9 & 2.1 & 145.7 & 122.9 & 3.288 & 0.000 & 2.1 & 2.1 & 122.6 & 92.0 & 3.129 & 0.001 & \\
\hline U5005 & 3.3 & 0.6 & 124.6 & 13.5 & 0.191 & 0.991 & 3.5 & 0.6 & 107.9 & 10.2 & 0.191 & 0.995 & \\
\hline U1230 & 12.1 & 2.1 & 86.4 & 6.2 & 1.143 & 0.328 & 13.0 & 2.6 & 79.5 & 6.5 & 1.244 & 0.262 & \\
\hline U731 & 18.6 & 1.9 & 53.1 & 2.8 & 0.684 & 0.740 & 20.2 & 2.2 & 48.9 & 2.6 & 0.760 & 0.668 & \\
\hline U4173 & 0.1 & - & 319.6 & - & 0.058 & 1.000 & 2.0 & 0.7 & 74.4 & 17.4 & 0.116 & 0.999 & \\
\hline U4325 & 0.1 & - & 3331.6 & - & 1.330 & 0.132 & 0.1 & - & 3203.9 & - & 1.132 & 0.257 & \\
\hline N1560 & 5.3 & 0.5 & 106.1 & 8.8 & 2.969 & 0.000 & 5.4 & 0.5 & 92.9 & 7.3 & 2.630 & 0.000 & \\
\hline U3371 & 0.1 & - & 875.6 & - & 0.287 & 0.996 & 0.1 & - & 814.7 & - & 0.273 & 0.997 & \\
\hline U628 & 12.9 & 1.2 & 101.2 & 5.7 & 0.347 & 0.912 & 12.9 & 1.2 & 101.2 & 5.7 & 0.346 & 0.912 & \\
\hline DDO185 & 0.1 & - & 784.5 & - & 2.677 & 0.005 & 0.1 & - & 651.1 & - & 2.326 & 0.012 & \\
\hline DDO189 & 9.9 & 1.3 & 59.2408 & 4.3 & 0.171 & 0.997 & 11.0 & 1.2 & 50.6 & 2.8 & 0.119 & 0.999 & \\
\hline DDO47 & 0.1 & - & 1332.5 & - & 0.320 & 0.897 & 0.1 & - & 1248.2 & - & 0.250 & 0.941 & \\
\hline DDO64 & 0.1 & - & 1182.3 & - & 0.838 & 0.539 & - & - & - & - & - & - & \\
\hline N2366 & 11.2 & 2.4 & 50.8487 & 7.5 & 2.154 & 0.001 & 12.8 & 2.8 & 41.1 & 5.8 & 2.240 & 0.000 & \\
\hline N3274 & 30.4 & 2.1 & 49.6296 & 1.6 & 0.894 & 0.600 & 31.0 & 2.2 & 47.7 & 1.5 & 0.830 & 0.685 & \\
\hline N4395 & 12.1 & 0.9 & 69.7218 & 3.8 & 0.762 & 0.898 & 13.2 & 1.0 & 63.3 & 3.2 & 0.733 & 0.927 & \\
\hline N4455 & 5.2 & 1.0 & 99.7369 & 19.3 & 0.452 & 0.976 & 5.4 & 1.0 & 86.8 & 14.7 & 0.393 & 0.990 & \\
\hline K124 & 0.2 & 6.0 & 661.1 & 4320.4 & 1.668 & 0.034 & - & - & - & - & - & - & \\
\hline N100 & 8.3 & 1.2 & 104.7 & 13.2 & 0.939 & 0.018 & - & - & - & - & - & - & \\
\hline U1281 & 0.1 & - & 785.0 & - & 1.376 & 0.080 & - & - & - & - & - & - & \\
\hline U3137 & 10.0 & 0.8 & 88.2 & 2.6 & 2.188 & 0.000 & - & - & - & - & - & - & \\
\hline DDO52 & 0.1 & - & 991.0 & - & 2.921 & 0.004 & - & - & - & - & - & - & \\
\hline IC2233 & 0.1 & - & 1135.0 & - & 1.256 & 0.145 & - & - & - & - & - & - & \\
\hline N5023 & 11.4 & 1.1 & 84.6 & 7.3 & 1.044 & 0.398 & - & - & - & - & - & - & \\
\hline \multirow[t]{2}{*}{ U10310 } & 2.6 & 2.5 & 181.3 & 160.9 & 0.590 & 0.873 & - & - & - & - & - & - & \\
\hline & \multicolumn{6}{|c|}{ Constant $M / L_{\star}$} & \multicolumn{7}{|c|}{ Maximum disk } \\
\hline F5631 & 6.8 & 1.6 & 103.5 & 12.9 & 0.360 & 0.904 & 1.6 & 2.8 & 220.3 & 282.0 & 0.373 & 0.895 & 5.9 \\
\hline U5750 & 0.8 & 3.6 & 187.7 & 474.3 & 3.110 & 0.000 & 0.6 & 4.3 & 215.5 & 772.3 & 3.118 & 0.000 & 1.6 \\
\hline U5005 & 2.3 & 0.6 & 127.2 & 19.7 & 0.170 & 0.997 & 0.1 & - & 307.1 & - & 0.557 & 0.475 & 5.4 \\
\hline U1230 & 11.7 & 2.4 & 77.7 & 6.6 & 1.091 & 0.365 & 6.6 & 2.0 & 73.0 & 8.7 & 0.724 & 0.687 & 6.1 \\
\hline U731 & 19.5 & 2.3 & 47.6 & 2.8 & 0.728 & 0.699 & 9.3 & 4.0 & 31.8 & 6.6 & 0.562 & 0.847 & 13.5 \\
\hline U4173 & 0.1 & - & 176.2 & - & 0.129 & 1.000 & 0.1 & - & 128.9 & - & 0.236 & 0.993 & 2.4 \\
\hline $\mathrm{U} 4325$ & 0.1 & - & 2655.1 & - & 1.157 & 0.237 & 0.1 & - & 1724.1 & - & 1.225 & 0.172 & 4.6 \\
\hline N1560 & 1.8 & 1.0 & 218.9 & 105.2 & 2.243 & 0.000 & 0.1 & - & 520.6 & - & 8.859 & 0.000 & 4.9 \\
\hline U3371 & 0.1 & - & 735.3 & - & 0.278 & 0.997 & 0.1 & - & 533.1 & - & 0.391 & 0.980 & 5.0 \\
\hline U628 & 12.0 & 1.3 & 97.2 & 6.3 & 0.341 & 0.915 & 0.1 & - & 273.5 & - & 0.619 & 0.689 & 9.8 \\
\hline DDO185 & 0.1 & - & 575.0 & - & 2.269 & 0.014 & 0.1 & - & 382.9 & - & 2.137 & 0.021 & 5.0 \\
\hline DDO189 & 9.6 & 1.1 & 52.3 & 3.3 & 0.099 & 1.000 & 1.5 & 3.0 & 148.6 & 220.0 & 0.156 & 0.998 & 8.3 \\
\hline DDO47 & 0.1 & - & 1178.0 & - & 0.307 & 0.905 & 0.1 & - & 1029.8 & - & 0.455 & 0.785 & 4.4 \\
\hline DDO64 & 0.1 & - & 966.0 & - & 0.770 & 0.615 & 0.1 & - & 562.1 & - & 0.739 & 0.651 & 4.1 \\
\hline N2366 & 11.9 & 3.0 & 36.4 & 5.8 & 1.932 & 0.003 & 8.6 & 7.3 & 14.9 & 7.0 & 1.245 & 0.181 & 5.4 \\
\hline N3274 & 23.7 & 2.1 & 50.4 & 2.2 & 0.928 & 0.554 & 12.6 & 2.6 & 61.8 & 7.7 & 1.804 & 0.013 & 3.9 \\
\hline N4395 & 12.6 & 1.1 & 58.0 & 3.1 & 0.646 & 0.979 & 11.9 & 1.2 & 50.8 & 3.1 & 0.577 & 0.994 & 3.0 \\
\hline N4455 & 0.1 & - & 708.3 & 32.1 & 0.630 & 0.849 & 0.1 & - & 602.4 & - & 1.495 & 0.056 & 2.3 \\
\hline
\end{tabular}


Table 5. Parameters pseudo-isothermal models.

\begin{tabular}{|c|c|c|c|c|c|c|c|c|c|c|c|c|c|}
\hline Name & $R_{\mathrm{C}}$ & $\Delta R$ & $\rho_{0}$ & $\Delta \rho$ & $\chi^{2}$ & $p$ & $R_{\mathrm{C}}$ & $\Delta R$ & $\rho_{0}$ & $\Delta \rho$ & $\chi^{2}$ & $p$ & $M / L_{\star}^{\max }$ \\
\hline & \multicolumn{6}{|c|}{ Minimum disk } & \multicolumn{6}{|c|}{ Minimum disk + gas } & \\
\hline F5631 & 2.0 & 0.2 & 70.4 & 13.1 & 0.203 & 0.976 & 1.9 & 0.2 & 73.3 & 14.9 & 0.217 & 0.971 & \\
\hline U5750 & 5.0 & 0.9 & 7.9 & 1.6 & 1.026 & 0.321 & 4.8 & 1.0 & 7.4 & 1.8 & 1.148 & 0.260 & \\
\hline U5005 & 4.7 & 0.2 & 11.5 & 0.8 & 0.062 & 1.000 & 4.2 & 0.2 & 11.7 & 0.9 & 0.047 & 1.000 & \\
\hline U1230 & 1.5 & 0.3 & 103.5 & 38.6 & 0.774 & 0.640 & 1.4 & 0.4 & 114.5 & 55.2 & 1.030 & 0.413 & \\
\hline $\mathrm{U} 731$ & 0.6 & 0.1 & 333.9 & 46.0 & 0.313 & 0.976 & 0.5 & 0.1 & 381.8 & 69.9 & 0.439 & 0.928 & \\
\hline U4173 & 3.5 & 0.2 & 7.3 & 0.7 & 0.066 & 1.000 & 2.6 & 0.2 & 7.9 & 0.8 & 0.041 & 1.000 & \\
\hline U4325 & 2.7 & 0.1 & 100.1 & 2.1 & 0.017 & 1.000 & 2.5 & 0.1 & 100.6 & 2.0 & 0.015 & 1.000 & \\
\hline N1560 & 1.6 & 0.1 & 57.1 & 4.3 & 4.343 & 0.000 & 1.5 & 0.1 & 54.6 & 4.8 & 4.306 & 0.000 & \\
\hline U3371 & 3.7 & 0.1 & 18.0 & 0.3 & 0.003 & 1.000 & 3.3 & 0.1 & 19.2 & 0.6 & 0.009 & 1.000 & \\
\hline U628 & 1.5 & 0.2 & 151.9 & 27.8 & 0.392 & 0.883 & 1.5 & 0.2 & 151.9 & 27.8 & 0.392 & 0.883 & \\
\hline DDO185 & $\infty$ & - & 22.4 & 2.0 & 0.669 & 0.672 & $\infty$ & - & 20.7 & 2.5 & 1.020 & 0.372 & \\
\hline DDO189 & 1.0 & 0.1 & 97.9 & 11.3 & 0.064 & 1.000 & 0.8 & 0.1 & 115.0 & 13.7 & 0.053 & 1.000 & \\
\hline DDO47 & 2.1 & 0.5 & 47.5 & 10.2 & 0.205 & 0.975 & 1.9 & 0.4 & 48.4 & 10.4 & 0.173 & 0.984 & \\
\hline DDO64 & 1.2 & 0.2 & 72.7 & 11.9 & 0.423 & 0.955 & 1.2 & 0.2 & 72.7 & 11.9 & 0.423 & 0.955 & \\
\hline N2366 & 0.7 & 0.1 & 147.3 & 26.4 & 1.043 & 0.402 & 0.6 & 0.1 & 154.7 & 37.2 & 1.316 & 0.129 & \\
\hline N3274 & 0.3 & 0.1 & 1259.4 & 255.6 & 0.918 & 0.565 & 0.3 & 0.1 & 1340.2 & 290.2 & 0.906 & 0.582 & \\
\hline N4395 & 0.9 & 0.1 & 175.6 & 18.9 & 0.601 & 0.991 & 0.8 & 0.1 & 202.0 & 21.8 & 0.513 & 0.999 & \\
\hline N4455 & 1.3 & 0.1 & 63.2 & 5.1 & 0.299 & 0.998 & 1.2 & 0.1 & 61.0 & 5.4 & 0.283 & 0.999 & \\
\hline $\mathrm{K} 124$ & 4.4 & 0.2 & 13.5 & 0.8 & 0.236 & 1.000 & - & - & - & - & - & - & \\
\hline N100 & 1.4 & 0.1 & 105.3 & 4.1 & 0.106 & 0.688 & - & - & - & - & - & - & \\
\hline U1281 & 2.2 & 0.1 & 28.0 & 1.7 & 0.169 & 1.000 & - & - & - & - & - & - & \\
\hline U3137 & 2.0 & 0.2 & 61.2 & 8.7 & 1.491 & 0.278 & - & - & - & - & - & - & \\
\hline DDO52 & 0.7 & 0.3 & 123.8 & 69.0 & 2.193 & 0.052 & - & - & - & - & - & - & \\
\hline IC2233 & 3.7 & 0.5 & 23.0 & 2.9 & 1.532 & 0.049 & - & - & - & - & - & - & \\
\hline N5023 & 0.9 & 0.1 & 200.7 & 10.7 & 0.277 & 1.000 & - & - & - & - & - & - & \\
\hline U10310 & 2.5 & 0.2 & 27.2 & 2.2 & 0.081 & 1.000 & - & - & - & - & - & - & \\
\hline \multicolumn{7}{|c|}{ Constant $M / L_{\star}$} & \multicolumn{6}{|c|}{ Maximum disk } & \\
\hline F5631 & 2.0 & 0.3 & 57.4 & 14.3 & 0.261 & 0.955 & 3.9 & 1.4 & 15.3 & 8.4 & 0.427 & 0.861 & 5.9 \\
\hline U5750 & 5.9 & 1.7 & 4.7 & 1.4 & 1.311 & 0.144 & 6.1 & 1.8 & 4.5 & 1.4 & 1.349 & 0.128 & 1.6 \\
\hline U5005 & 5.4 & 0.3 & 7.4 & 0.5 & 0.041 & 1.000 & 16.0 & 4.2 & 1.5 & 0.3 & 0.150 & 0.998 & 5.4 \\
\hline U1230 & 1.5 & 0.4 & 90.2 & 43.1 & 0.825 & 0.593 & 2.2 & 0.7 & 27.8 & 14.9 & 0.452 & 0.907 & 6.1 \\
\hline U731 & 0.5 & 0.1 & 346.4 & 68.0 & 0.433 & 0.931 & 0.7 & 0.3 & 63.4 & 42.7 & 0.476 & 0.906 & 13.5 \\
\hline U4173 & 4.2 & 0.6 & 3.2 & 0.5 & 0.062 & 1.000 & 7.0 & 2.2 & 1.4 & 0.4 & 0.118 & 1.000 & 2.4 \\
\hline $\mathrm{U} 4325$ & 3.2 & 0.1 & 72.2 & 1.1 & 0.006 & 1.000 & 9.1 & 4.7 & 35.2 & 2.4 & 0.076 & 1.000 & 3.8 \\
\hline N1560 & 2.1 & 0.2 & 29.4 & 2.9 & 4.298 & 0.000 & 13.2 & 4.2 & 4.8 & 0.4 & 2.445 & 0.000 & 4.9 \\
\hline U3371 & 3.7 & 0.1 & 14.7 & 0.7 & 0.016 & 1.000 & 5.9 & 0.8 & 6.5 & 0.8 & 0.080 & 1.000 & 5.0 \\
\hline U628 & 1.5 & 0.2 & 132.0 & 29.3 & 0.447 & 0.847 & 0.4 & 0.8 & 223.6 & 893.1 & 0.946 & 0.460 & 9.8 \\
\hline DDO185 & $\infty$ & - & 16.9 & 2.4 & 1.043 & 0.392 & $\infty$ & - & 11.6 & 2.7 & 1.321 & 0.257 & 5.0 \\
\hline DDO189 & 0.9 & 0.1 & 88.6 & 10.3 & 0.042 & 1.000 & 2.8 & 0.4 & 11.9 & 2.4 & 0.047 & 1.000 & 8.3 \\
\hline DDO47 & 2.4 & 0.7 & 37.6 & 7.8 & 0.152 & 0.989 & 7.7 & 9.1 & 21.3 & 3.5 & 0.101 & 0.996 & 4.4 \\
\hline DDO64 & 1.4 & 0.3 & 52.7 & 10.1 & 0.413 & 0.959 & 2.7 & 1.9 & 22.0 & 6.5 & 0.439 & 0.948 & 4.1 \\
\hline N2366 & 0.6 & 0.1 & 126.0 & 37.5 & 1.258 & 0.171 & 0.5 & 0.4 & 35.5 & 46.5 & 1.165 & 0.256 & 5.4 \\
\hline N3274 & 0.4 & 0.1 & 585.3 & 120.7 & 0.787 & 0.738 & 1.0 & 0.2 & 132.0 & 33.2 & 1.064 & 0.376 & 4.0 \\
\hline N4395 & 0.8 & 0.1 & 184.2 & 23.2 & 0.478 & 1.000 & 0.7 & 0.1 & 167.1 & 27.0 & 0.454 & 1.000 & 3.0 \\
\hline N4455 & 2.4 & 0.2 & 22.6 & 2.0 & 0.225 & 1.000 & 4.0 & 0.7 & 12.4 & 1.7 & 0.400 & 0.988 & 2.3 \\
\hline
\end{tabular}



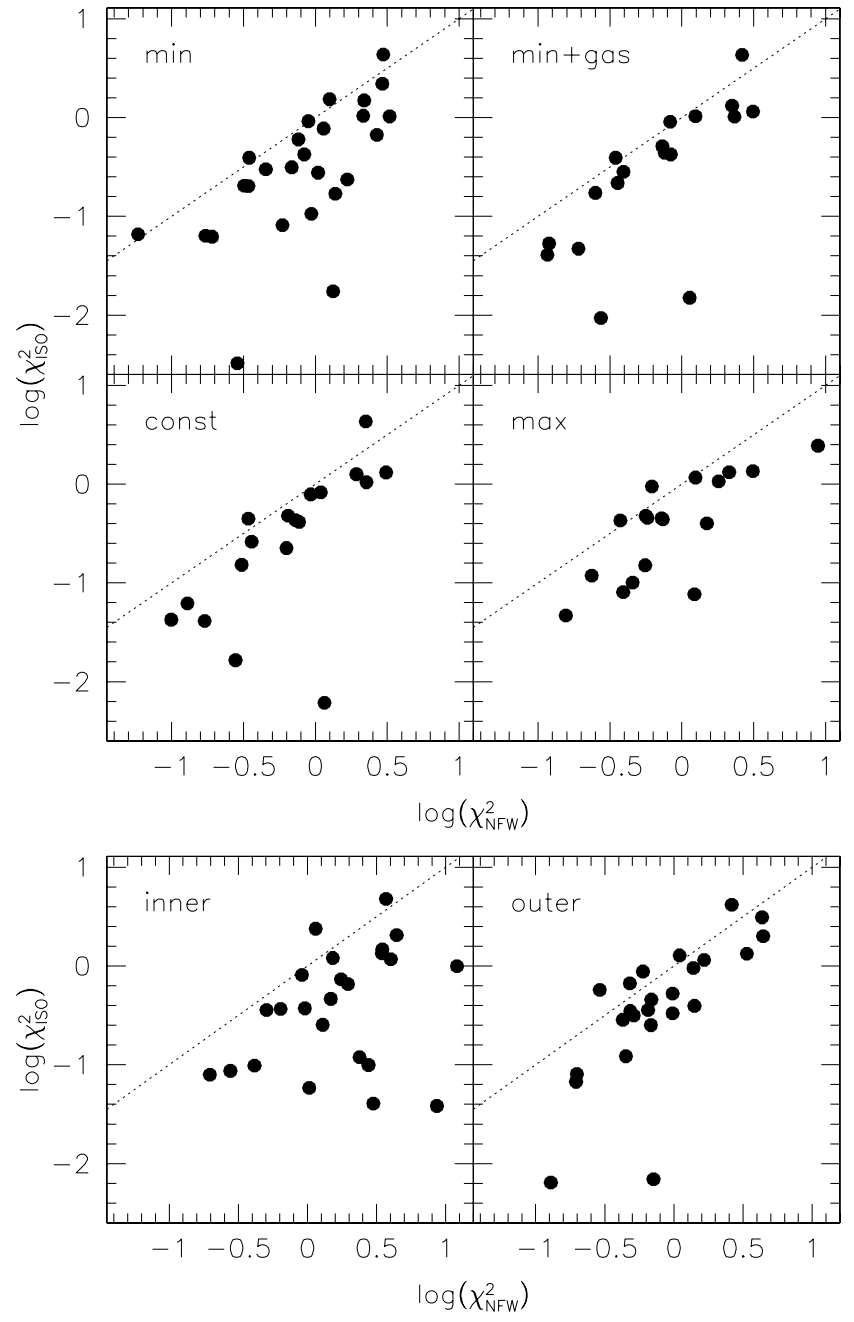

Fig. 9. Top: Comparison of the reduced $\chi^{2}$ values of massmodels using the NFW and the pseudo-isothermal halos. In all cases the pseudo-isothermal fits are equal to or better than the NFW fits. Dotted lines are lines of equality. Bottom: Comparison of the reduced $\chi^{2}$ values for the minimum disk case. Left panel compares the values for the inner half of the rotation curve, right panel compares the outer half of the rotation curve. In both cases one sees that pseudo-isothermal halos fit better.

We note that the $\chi^{2}$ values given here should not be regarded as absolute. As described in Sect. 5, the combination of $\mathrm{H} \alpha$ and $\mathrm{HI}$ data, the symmetrization of the curves, the definition of the subsequent error and the imposition of a minimum error result in a conservative estimate of the uncertainty. The errorbars we give here are likely an overestimate of the true uncertainty. This at least partly explains the very low $\chi^{2}$ values found for some curves.

The bottom panel in Fig. 9 compares the $\chi^{2}$ values for the minimum disk case, but measured separately for the inner and outer halves of the rotation curves, respectively. The largest discrepancies between pseudo-isothermal and NFW are found in the inner parts, as expected, but it is clear that also in the outer parts pseudo-isothermal models generally provide better fits. It is remarkable that despite the large errorbars, there is still an obvious preference for the pseudo-isothermal model. This conclusion is indepen-
Table 6. Comparison NFW/ISO.

\begin{tabular}{lllll}
\hline & \multicolumn{2}{c}{ NFW } & \multicolumn{2}{c}{ ISO } \\
& $p>0.95$ & $p<0.05$ & $p>0.95$ & $p<0.05$ \\
\hline min & 5 & 4 & 11 & 0 \\
min+gas & 5 & 5 & 10 & 0 \\
con & 5 & 4 & 10 & 0 \\
$\max$ & 5 & 2 & 8 & 0 \\
\hline
\end{tabular}

NGC 1560 has been excluded from this analysis due to the non-representative $p$-values.

dent of any over- or underestimate of the true error: a less conservative estimate would even strengthen the trends observed here.

The pseudo-isothermal model has no bad $(p<0.05)$ fits, in contrast with NFW. The absolute number of good fits is a factor 2 larger for the pseudo-isothermal model. The proportion of "average" fits $(0.05 \leq p \leq 0.95)$ is larger in the NFW case. The conclusion is that the pseudoisothermal halo gives a better description of the data.

\subsection{NFW halos}

The mass and concentration of (numerically simulated) CDM halos depend on the cosmological assumptions that are used as input to the simulations. NFW halos follow a specific relation between $c$ and $V_{200}$ (Navarro et al. 1997). Independent simulations have also given a reasonable idea of the scatter one expects at fixed halo mass (Jing 2000; Bullock et al. 2001), even though they do not agree on the details. In Fig. 10 we compare our results with these predictions.

Shown as the full line is the prediction for a $\Lambda$ dominated flat universe $\left(\Omega_{\mathrm{m}}=0.3, \Omega_{\Lambda}=0.7\right)$. There is a general trend of increasing $c$ values towards lower $V_{200}$. There is some disagreement regarding the theoretical scatter in the $c$-values. Bullock et al. (2001) quotes a scatter in $\log c$ of 0.18, while Jing (2000) derives a smaller scatter in $\log c$ of 0.08. Here we adopt the larger value from Bullock et al. (2001) in order to give the CDM models as much leeway as possible. The dotted lines in Fig. 10 indicate the $1 \sigma$ (grey area) and $2 \sigma$ scatter where $\sigma=\sigma(\log c)=0.18$. The small dots represent the values for other LSB and dwarf galaxies derived in dBMR. Our data show a similar behaviour, though the locus of our data seems to be better defined than that of the dBMR data. The spread in $c$ larger than the models predict, which is best illustrated by the excess of low- $c$ points.

\section{Pointing effects}

In an analysis of this nature it is crucial that the slit is aligned with the center of the galaxy. Any off-set from center will lead to a rotation curve that is less steep than a properly centered rotation curve unless the entire galaxy is a pure solid body rotator. It is therefore theoretically conceivable that systematic effects may have caused us to underestimate the inner slope of the rotation curve, thus 

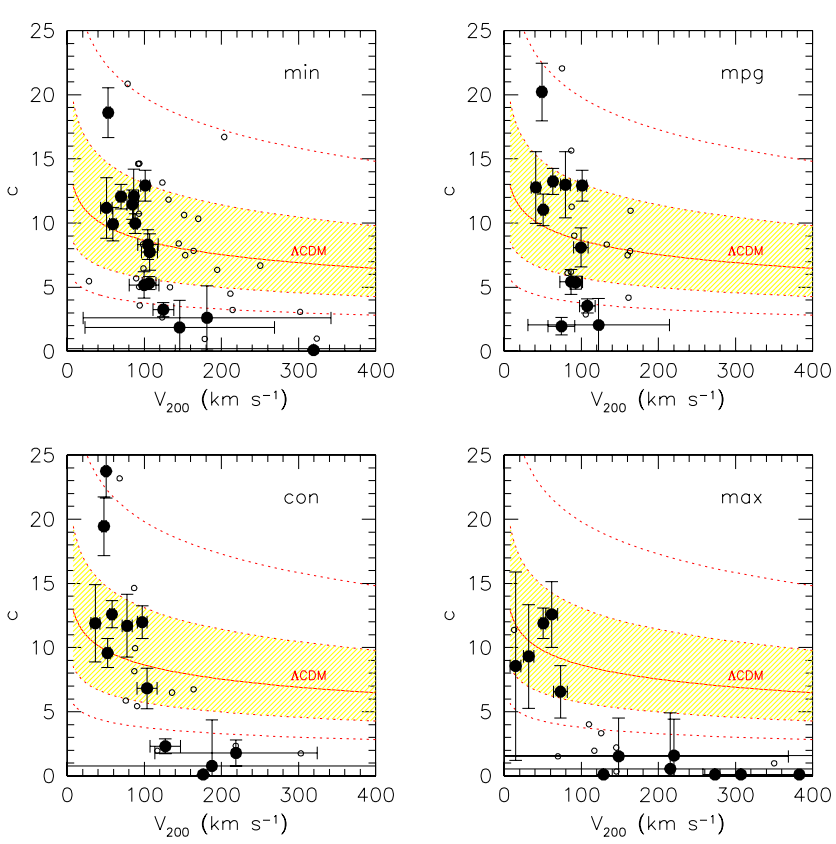

Fig. 10. The NFW halo concentration parameter $c$ plotted against the halo rotation velocity $V_{200}$ for the four different $M / L_{\star}$ cases discussed in this paper. Large black dots are represent our sample. For comparison, small open dots indicate the galaxies from the dBMR sample. The line labelled " $\Lambda \mathrm{CDM}$ " shows the prediction for that cosmology derived from numerical models. The grey area encloses the $1 \sigma$ uncertainty (Bullock et al. 1999). The upper and lower dotted line show the $2 \sigma$ uncertainty.

mistaking the incorrectly lowered slope of the NFW profile for that of an isothermal halo.

Here we present several arguments why this is not the case, and why the observationally derived slopes are very close to the true slopes.

\subsection{Evidence from observations}

As already mentioned in Sect. 2, we took considerable care when acquiring the galaxy at the telescope. Most of the nearby galaxies in our sample were visible in the guidingcamera, and they were used to test our off-set procedure. This involved re-acquiring a galaxy several times in order to test the stability of the procedure, as well as moving back and forth to the acquisition star to test for repeatability. We found the stability of the system to be better than $1^{\prime \prime}$. Of course, for the faintest galaxies, we rely entirely on the position given for the center, which we took from Swaters (1999) or Stil (1999) or the NED database.

There are no systematic differences between the rotation curves acquired by us at the $1.93 \mathrm{~m}$ telescope at OHP, by McGaugh et al. (2001) at the Kitt-Peak $4 \mathrm{~m}$ telescope, and the Las Campanas du Pont 2.5 telescope, by Swaters et al. (2000) at the 200" Hale Telescope at Mt. Palomar and by Pickering et al. (1998) at the MMT. This indicates that either all or none of the data sets suffer from systematic effects due to mispointing. A number of galaxies have been observed multiple times by different observers, and similarly show no systematic differences. This is illustrated
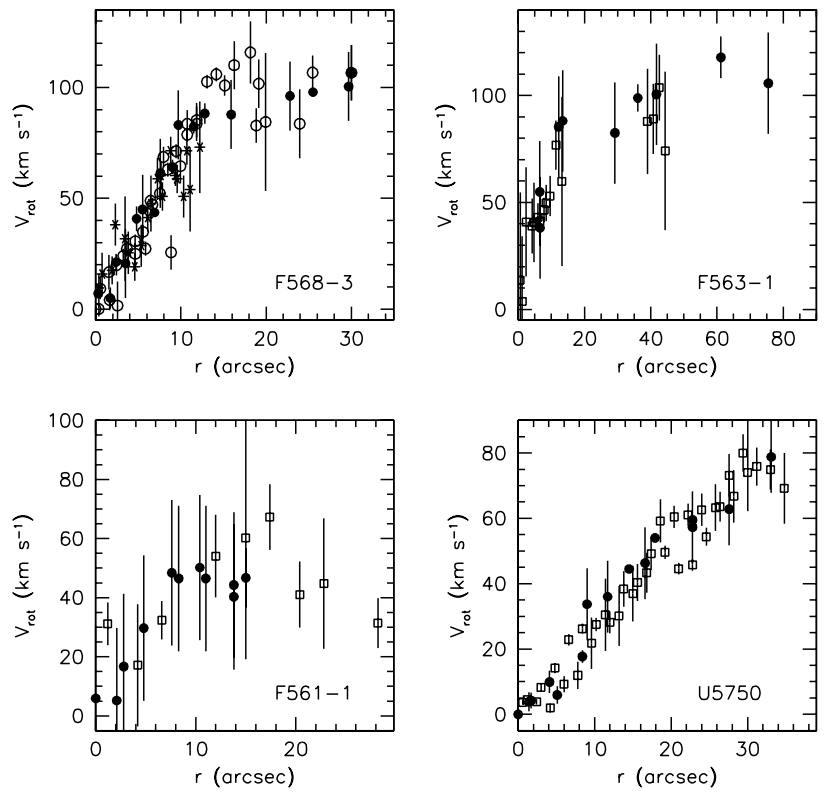

Fig. 11. The rotation curves derived from independent $\mathrm{H} \alpha$ observations for four LSB galaxies. Open squares are the data presented here. Solid circles are from McGaugh et al. (2001). Open circles in the top-left panel (F568-3) are from Swaters et al. (2000) and stars are from Pickering et al. (1998). Agreement between independent observations in good.

in Fig. 11 where the raw data of four LSB galaxy rotation curves are compared. In one case there are three independent data sets available, all agreeing with each other. It is unlikely that independent observers miss the center of the galaxy by the same amount in the same direction every time.

If the true rotation curves were really bona-fide NFW curves we would expect that with the increased resolution of the $\mathrm{H} \alpha$ data, at least some of them should start to look more like NFW curves. After all, the combined samples in this paper and McGaugh et al. (2001) contain a few dozen rotation curves. Even in the presence of (hypothetical) pointing effects, some should have hit the nucleus and have shown the characteristic NFW shape. This has not happened. The situation is quite the reverse: the best resolved rotation curves show the flattest cores (see Sect. 9 and de Blok et al. 2001b).

This leads to an interesting contradiction: if indeed we imagine that a pointing offset leads to a systematic under estimate of the slope, we ought to see a trend of rotation curve shape with distance. This is not seen. A pointing offset of a given number of arcseconds corresponds to a different physical scale in each galaxy, depending on its distance. Our sample and that of McGaugh et al. (2001) span a range in distance of a factor of $\sim 200$. If we imagine that a pointing offset of, say, $0.5^{\prime \prime}$ causes us to mistake an NFW halo for a pseudo-isothermal one in the distant galaxies in our sample, then this implies that in order to mislead ourselves in a similar way, we ought to be making an error of almost $1.5^{\prime}$ in our pointing when observing the nearest galaxies. Though this is an extreme example, it is highly improbably that systematic effects in target 
acquisition at the telescope depend on the distance of the target.

Assuming then that we managed in the large majority of the cases to indeed home in on the center of the light distribution of the galaxy, one could still assume that the dynamical center of the galaxy does not coincide with the center of the light distribution. Centering on the light distribution would thus not give us the true rotation curve. This has of course just the same effect of mis-pointing, but now the fault lies with the galaxy, rather than the observer.

To test this one needs high-resolution velocity fields, to which one can then fit a tilted ring model. The tilted ring model has the central position of the rings as a free parameter, and will thus immediately show whether there is a significant discrepancy between the optical and the dynamical center or not. This is precisely what BlaisOuellette et al. (2001) have done. They have derived $\mathrm{H} \alpha$ rotation curves for a number of late-type galaxies using full 2D Fabry-Perot velocity fields. They find the same slowlyrising rotation curves, and conclude that NFW models are incompatible with their data, which prefer a coredominated halo or a model with a shallow inner density slope.

This is the same conclusion as reached by Bolatto et al. (2002), who obtained high-resolution 5" CO observations of the nearby dwarf galaxy NGC 4605. They derived a rotation curve from a $3 \mathrm{D}$ data cube, again using a tilted ring model, and find a slowly rising rotation curve incompatible with NFW.

A number of galaxies in our sample is edge-on, and it is conceivable that, despite contrary evidence, these data are affected by optical depth or projection effects. These edge-on galaxies are however only a small fraction of the galaxies investigated. For example, of the 56 galaxies presented here and in de Blok et al. (2001a), only 16 have inclinations larger than $70^{\circ}$. Though there are no systematic differences between the $\sim 30$ per cent with $i \geq 70^{\circ}$ and the $\sim 70$ per cent with $i<70^{\circ}$, one could easily disregard the high-inclination galaxies without affecting any of the conclusions. There are no significant trends of $c$ or slope with inclination.

In summary, there is no evidence that the data set presented in this paper suffers from significant systematic effects, nor is it inconsistent with any other relevant data set in the literature. Pointing effects play no significant role, and the observed rotation curves represent the overall dynamics of our galaxies well.

\subsection{Evidence from models}

To quantify the effects of mispointing we have created model NFW velocity fields and have derived rotation curves for various pointing offsets. We consider model velocity fields assuming NFW halos with $i=60^{\circ}, V_{200}=$ $100 \mathrm{~km} \mathrm{~s}^{-1}$ and $c=4,9,20$, respectively. Each of these three velocity fields is "observed" with a 1 " wide slit, off-
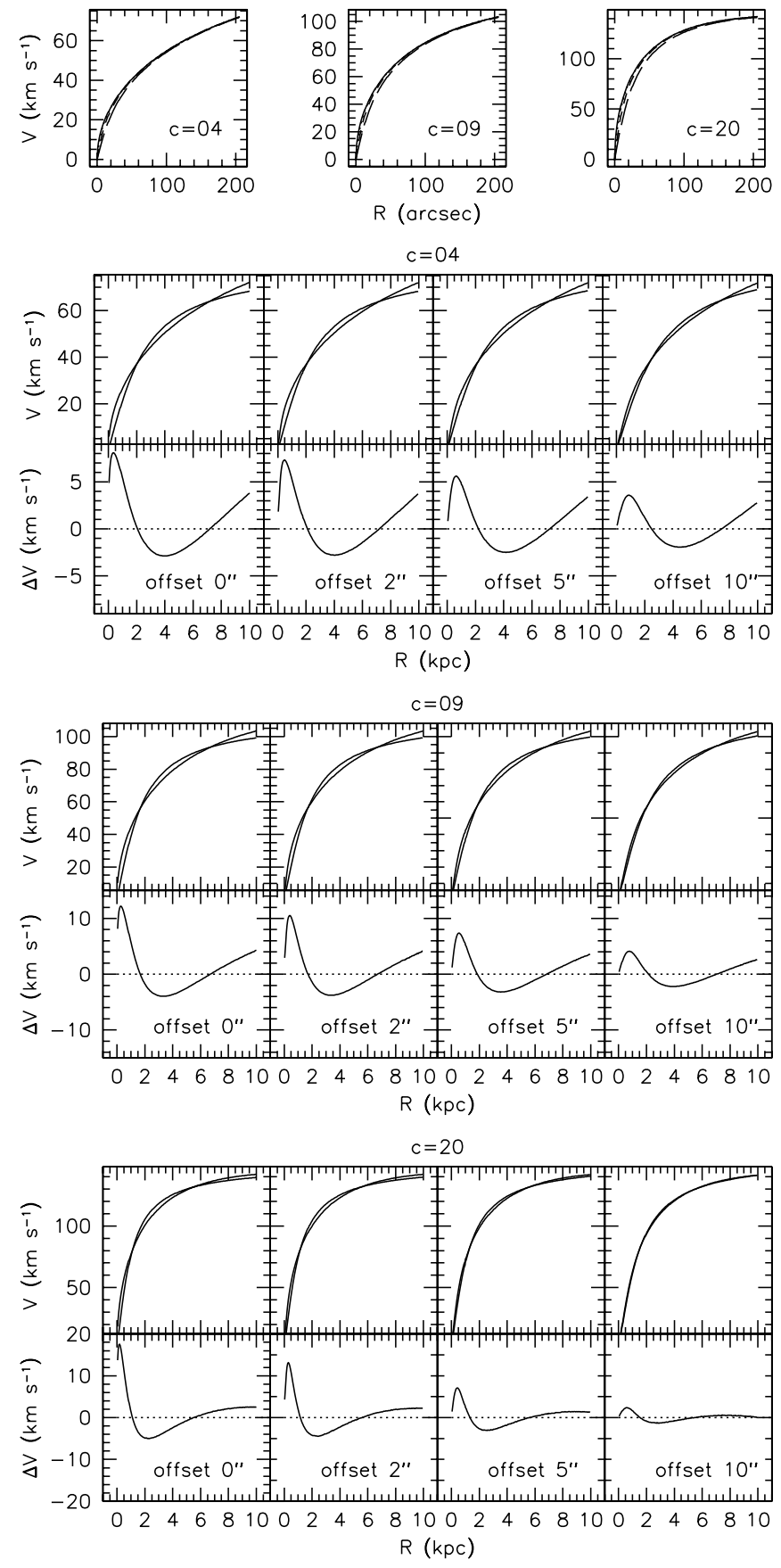

Fig. 12. Top panel: NFW model curves assuming $D=10 \mathrm{Mpc}$, $V_{200}=100 \mathrm{~km} \mathrm{~s}^{-1}$, and $c=4$ (left), 9 (middle) and 20 (right). For each $c$ we derived curves for various offsets: $0^{\prime \prime}$ (full line), $2^{\prime \prime}$ (dotted), $5^{\prime \prime}$ (short-dashed) and 10" (long-dashed). The other panels show the rotation curves for each combination of $c$ and offset (thick curve). Overplotted are the best fitting pseudoisothermal models (thin curve). Also shown are the differences between the two models.

set by $d=0^{\prime \prime}, 2^{\prime \prime}, 5^{\prime \prime}, 10^{\prime \prime}$ from the center, parallel to the major axis. We assume a distance of $10 \mathrm{Mpc}$, which is the average distance of the large majority of galaxies in our sample (disregarding the 4 galaxies with $D>40 \mathrm{Mpc}$.)

The top panel in Fig. 12 shows the rotation curves, for each combination of $c$ and $d$. The most important question we want to answer here is under what conditions an NFW 
velocity field can produce a rotation curve that mimics a pseudo-isothermal curve.

The three bottom panels in Fig. 12 show the derived offset NFW curves, with the best fitting pseudo-isothermal models overplotted. Also shown are the differences between both types of curves. It is clear that in almost all cases the shape of the pseudo-isothermal halo curve is distinctly different from that of the NFW curve. The only cases where one might mistake an offset NFW curve for a pseudo-isothermal curve are those for $\left(c=9 ; d=10^{\prime \prime}\right)$ and $\left(c=20 ; d=5^{\prime \prime}, 10^{\prime \prime}\right)$. Pointing errors of this magnitude are simply not present in the data. If we accept an uncertainty of at most a few tenths of an arcsecond, it is easy to see that pointing effects are only likely to affect galaxies at distances $\gtrsim 100 \mathrm{Mpc}$.

We can also regard the NFW curves in Fig. 12 as best fits to the plotted pseudo-isothermal curves. In this case we can make a direct comparison with the fits plotted in Fig. 8, and see that the characteristic overprediction of the inner part of the curve is also present in the data. Except for the three cases mentioned above, Fig. 12 shows that in all cases the difference between NFW and pseudoisothermal is most pronounced in the inner $2 \mathrm{kpc}$ with residuals between $\sim 5$ and $\sim 20 \mathrm{~km} \mathrm{~s}^{-1}$. The majority of galaxies in Fig. 8 does show such residuals, meaning that even in the presence of (hypothetical) modest pointing offsets, NFW curves would look different from the curves observed and shown in Fig. 8.

In summary, for the galaxies in this sample the effect of missing the centers of the galaxies would not be strong enough to masquerade NFW curves as pseudo-isothermal curves, except in the case of very (unrealistically) large offsets $\left(\gtrsim 5^{\prime \prime}\right)$. This conclusion, combined with the conclusion from Sect. 8.1 that any pointing errors must be below the $1^{\prime \prime}$ level, shows that the observed rotation curves must be close to the true rotation curves. Systematic observational effects cannot hide NFW halos in LSB galaxies.

\section{Mass profiles}

\subsection{Introduction}

In de Blok et al. (2001b) (hereafter dBMBR) the rotation curve sample presented in dBMR and part of the sample (the Jan00 galaxies) presented in this work were used to calculate the mass-density profiles that give rise to the observed rotation curves. As the different halo models predict different shapes of the mass distribution this gives one a direct test of the applicability of a particular model.

The mass density profile is derived by assuming a spherical mass distribution. Then, from $\nabla^{2} \Phi=4 \pi G \rho$ and $\Phi=-G M / r$ one can derive the mass density $\rho(r)$ :

$4 \pi G \rho(r)=2 \frac{v}{r} \frac{\partial v}{\partial r}+\left(\frac{v}{r}\right)^{2}$.

In the above $v$ is the rotation velocity and $r$ is the radius.

This inversion is only valid if the contribution by the gas and stars is negligible, i.e. one implicitly assumes a situation (close to) minimum disk. It is well-established that this is a reasonable assumption for LSB (dwarf) galaxies. A minimum disk also provides an upper limit on the steepness of the slopes of the halo mass-density profiles: inclusion of gas and stars will necessarily tend to flatten the slopes.

dBMBR find that the shape of the minimum-disk halo profiles can usually be characterised by two power-law components of the form $\rho \sim r^{\alpha}$. The outer slope has a value close to $\alpha=-2$, whereas the inner slope is more shallow. The models tested here make distinct predictions regarding the value of the inner slope: the pseudoisothermal halo predicts $\alpha=0$, the NFW halo predicts $\alpha=-1$, and more recent CDM simulations (Moore et al. 1999) find even steeper values of $\alpha=-1.5$.

dBMBR find that the distribution of the inner slopes $\alpha$ is asymmetric, with a well-defined peak at $\alpha=-0.2 \pm 0.2$, inconsistent with CDM predictions. This distribution has a broad wing however, extending to values of $\alpha<-1.8$, seemingly consistent with the steep slopes demanded by CDM. dBMBR show that the inner slope one derives depends on the radius of the innermost sampled point $r_{\text {in }}$ of the rotation curve. Larger values of $r_{\text {in }}$ sample the range of radii where the NFW profile and the pseudo-isothermal profile have similar slopes (which does not imply they have similar shapes!). Small values of $r_{\text {in }}$ probe the region where the predicted slopes are distinctly different. The galaxies with small values for $r_{\text {in }}$ indeed show the clearest evidence for the presence of a constant-density core (i.e. $\alpha \sim 0$ ). dBMBR conclude that all data are consistent with LSB galaxies having core-dominated halos with core-radii of a few kpc.

The implication is that galaxies with large values of $r_{\text {in }}$ and consequently with steep slopes, should show much shallower slopes once the resolution is increased (and $r_{\text {in }}$ decreased). The current data set puts us in an excellent position to test this prediction. For a large number of galaxies we now have high-resolution rotation curves, as well as independent lower-resolution curves from Swaters (1999). We should thus see a systematic decrease in inner slope when moving from the pure Hi data to the high-resolution data.

\subsection{Results}

\subsubsection{Minimum disk profiles}

Figure 13 plots the derived mass profiles for our complete sample. It is clear that most of the galaxies from our Feb. 01 run, which were specifically chosen to be nearby objects, are also characterised by an almost flat inner core with a radius of a few $\mathrm{kpc}$, in contrast with the steep $\alpha=-1.5$ power-law slope demanded by CDM, or even the inner $\alpha=-1.0$ power-law slope of the NFW profile.

Table 7 lists the values of the slopes derived using the method described in dBMBR. In short, after determining the "break-radius" where the slope changes most rapidly, we determined the slope of the inner component using a weighted least-squares fit. The uncertainty was 


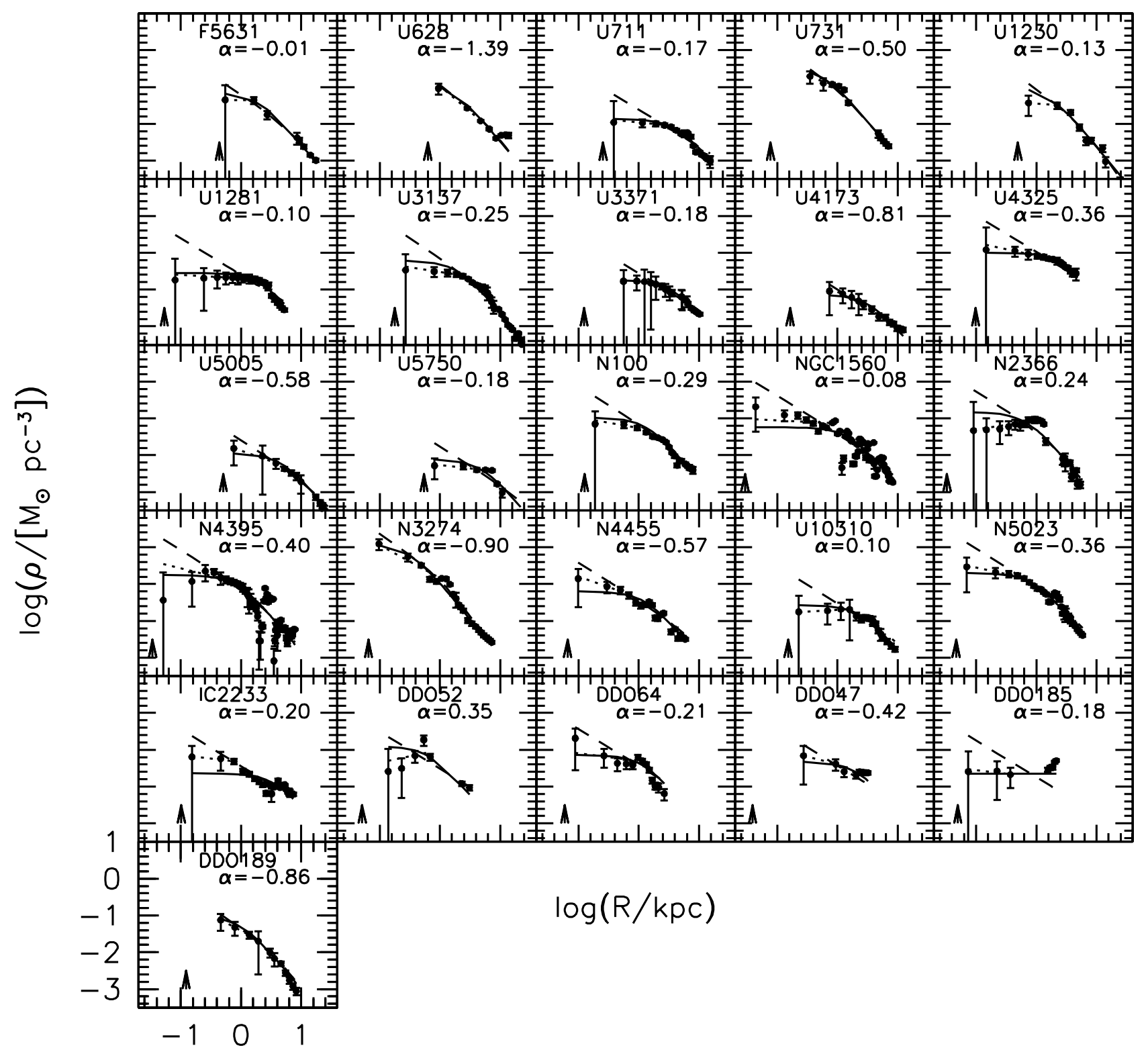

Fig. 13. Mass profiles of our sample galaxies derived from the high-resolution rotation curves. The profiles can be characterised by a steep $r^{-2}$ outer component, and a more shallow inner component ("core"). Also shown are the mass density profiles implied by the best-fitting minimum disk models. Shown are the pseudo-isothermal halo (full line) and the NFW halo (long-dashed line). We have also fitted a power-law to the inner shallow part (thick short-dashed line). The slope $\alpha$ is given in the top-left corners of the panels. All panels are at the same scale (denoted in bottom-left corner). Galaxies are labelled with their name. The arrows indicate an angular size of $2^{\prime \prime}$, the typical value of the seeing.

determined by re-measuring the slope twice, once by including the first data point outside the break-radius, and once by omitting the data point at the break-radius. The maximum difference between these two values and the original slope was adopted as the uncertainty. Following dBMBR we re-plot the values of the inner slope against the value of $r_{\text {in }}$, this time also including the Feb. 01 data (see Fig. 14). The new galaxies are consistent with a core, not a cusp, and the galaxies with small values of $r_{\text {in }}$ have flat mass density slopes.

\subsubsection{The case of N3274}

The only exception seems to be N3274 which shows a slope close to -1 even though its value for $r_{\text {in }}$ is quite small. The pseudo-isothermal halo fit shows that this galaxy has the smallest (minimum disk) core-radius and highest central density of the entire sample. Though seemingly consistent with the NFW profile, it is also consistent with a pseudo-isothermal halo profile with a small core radius. Furthermore, it has by far the highest surface brightness and smallest scale-length of the entire sample, and in this case it is very likely that the minimum disk assumption 


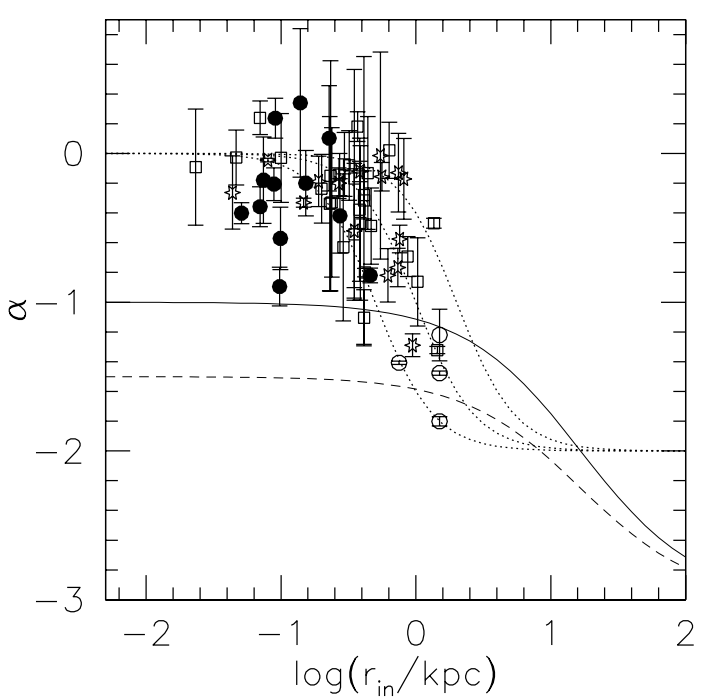

Fig. 14. Value of the inner slope $\alpha$ of the mass-density profiles plotted against the radius of the innermost point. Open circles are from the dBMR sample, stars are the Jan. 00 part of our sample, as published in dBMBR. Filled circles indicate the new galaxies from the Feb. 01 part of the sample. Over-plotted are the theoretical slopes of a pseudo-isothermal halo model (dotted lines) with core radii of 0.5 (left-most), 1 (centre) and 2 (right-most) kpc. The full line represents a NFW model, the dashed line a CDM $r^{-1.5}$ model. Both of the latter models have parameters $c=8$ and $V_{200}=100 \mathrm{~km} \mathrm{~s}^{-1}$, which were chosen to approximately fit the data points in the lower part of the diagram.

breaks down and the stellar component is important in the inner parts.

\subsubsection{Constant $M / L_{\star}$ profiles}

We tested this by re-deriving the slopes for the constant $M / L_{\star}=1.4$ case (see dBMR for a justification of this value). The halo rotation curve was found by quadratically subtracting the gas-rotation curve and the rotation curve of the stars. This treatment is likely to be too naive, as in a non-minimum disk case one expects the disk to influence the dark matter distribution to some degree (though perhaps not for LSB galaxies). A full treatment of this problem is beyond the scope of this paper, and the naive procedure suffices to illustrate the main point: as the stellar mass-to-light ratio is increased the inner slope of the halo mass-density profile becomes flatter.

The slopes for the constant $M / L_{\star}$ case are listed in Table 7. In Fig. 15 we again plot the derived slopes against the inner radii for the galaxies in our sample where a constant $M / L_{\star}$ model was available. A comparison with Fig. 14 shows that the data points have all moved up, as expected. In a number of cases we found rather large positive slopes, which would imply that these galaxies have hollow halos. This is rather improbable, and a more realistic explanation is that the value $M / L_{\star}=1.4$ is an overestimate of the true $M / L_{*}$.
Table 7. Inner power-law slopes $\alpha$.

\begin{tabular}{lrrrrr}
\hline Name & $\alpha_{\min }$ & $\Delta \alpha$ & $r_{\text {in }}(\mathrm{kpc})$ & $\alpha_{\text {con }}$ & $\Delta \alpha$ \\
\hline F563-1 & -0.01 & 0.70 & 0.55 & 0.21 & 1.38 \\
U628 & -1.29 & 0.08 & 0.95 & -1.37 & 1.37 \\
U711 & -0.12 & 0.07 & 0.38 & - & - \\
U731 & -0.52 & 0.45 & 0.35 & -0.44 & 0.15 \\
U1230 & -0.13 & 0.26 & 0.74 & 0.08 & 0.47 \\
U1281 & -0.04 & 0.01 & 0.08 & - & - \\
U3137 & -0.20 & 0.10 & 0.27 & - & - \\
U3371 & -0.16 & 0.10 & 0.56 & -0.02 & 0.19 \\
U4173 & -0.77 & 0.13 & 0.73 & -0.26 & 0.46 \\
U4325 & -0.33 & 0.03 & 0.15 & -0.24 & 0.06 \\
U5005 & -0.58 & 0.09 & 0.76 & -0.53 & 0.24 \\
U5750 & -0.17 & 0.27 & 0.81 & 0.26 & 0.71 \\
N100 & -0.19 & 0.17 & 0.19 & - & - \\
N1560 & -0.26 & 0.26 & 0.04 & -0.04 & 0.24 \\
N2366 & 0.24 & 0.13 & 0.09 & 0.45 & 0.45 \\
N4395 & -0.40 & 0.07 & 0.05 & -0.52 & 0.02 \\
N3274 & -0.90 & 0.13 & 0.10 & -0.67 & 0.17 \\
N4455 & -0.57 & 0.21 & 0.10 & -0.70 & 0.25 \\
U10310 & 0.10 & 0.36 & 0.22 & - & - \\
N5023 & -0.39 & 0.14 & 0.07 & - & - \\
IC2233 & -0.20 & 0.22 & 0.15 & - & - \\
DDO52 & 0.34 & 0.50 & 0.14 & - & - \\
DDO64 & -0.21 & 0.11 & 0.09 & -0.16 & 0.58 \\
DDO47 & -0.42 & 0.25 & 0.27 & -0.28 & 0.30 \\
DDO185 & -0.18 & 0.29 & 0.07 & -0.23 & 0.61 \\
DDO189 & -0.82 & 0.05 & 0.46 & -0.87 & 0.35 \\
\hline
\end{tabular}

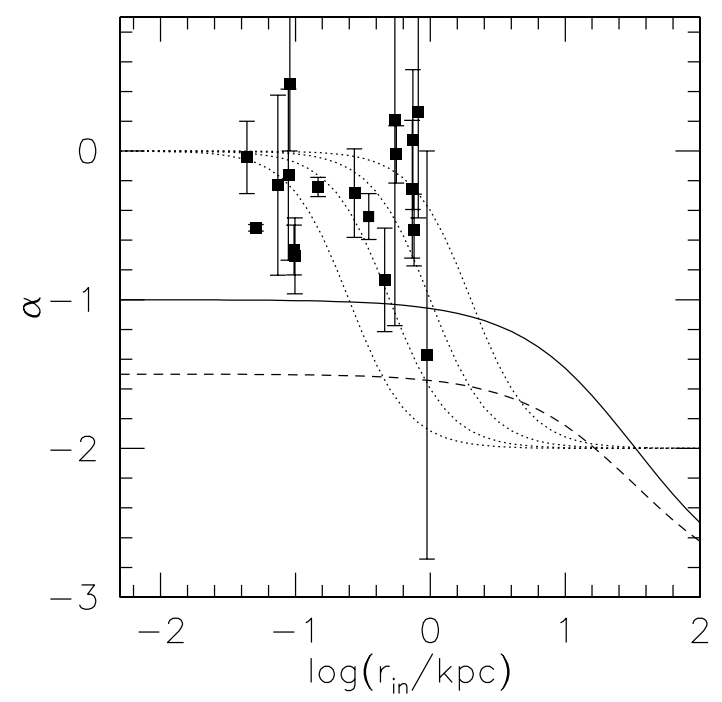

Fig. 15. The inner power-law slopes $\alpha$ for the constant $M / L_{*}=1.4$ assumption.

N3274 has a slope of -0.66 in the constant $M / L_{\star}$ case, which is consistent with the slope one would expect for a halo with a core-radius of a few tenths of a kpc. Though this galaxy obviously cannot be used to prove or disprove either model, it is clear that galaxies which show steep inner slopes are likely to be of high surface brightness with inner regions dominated by stars. In order to 


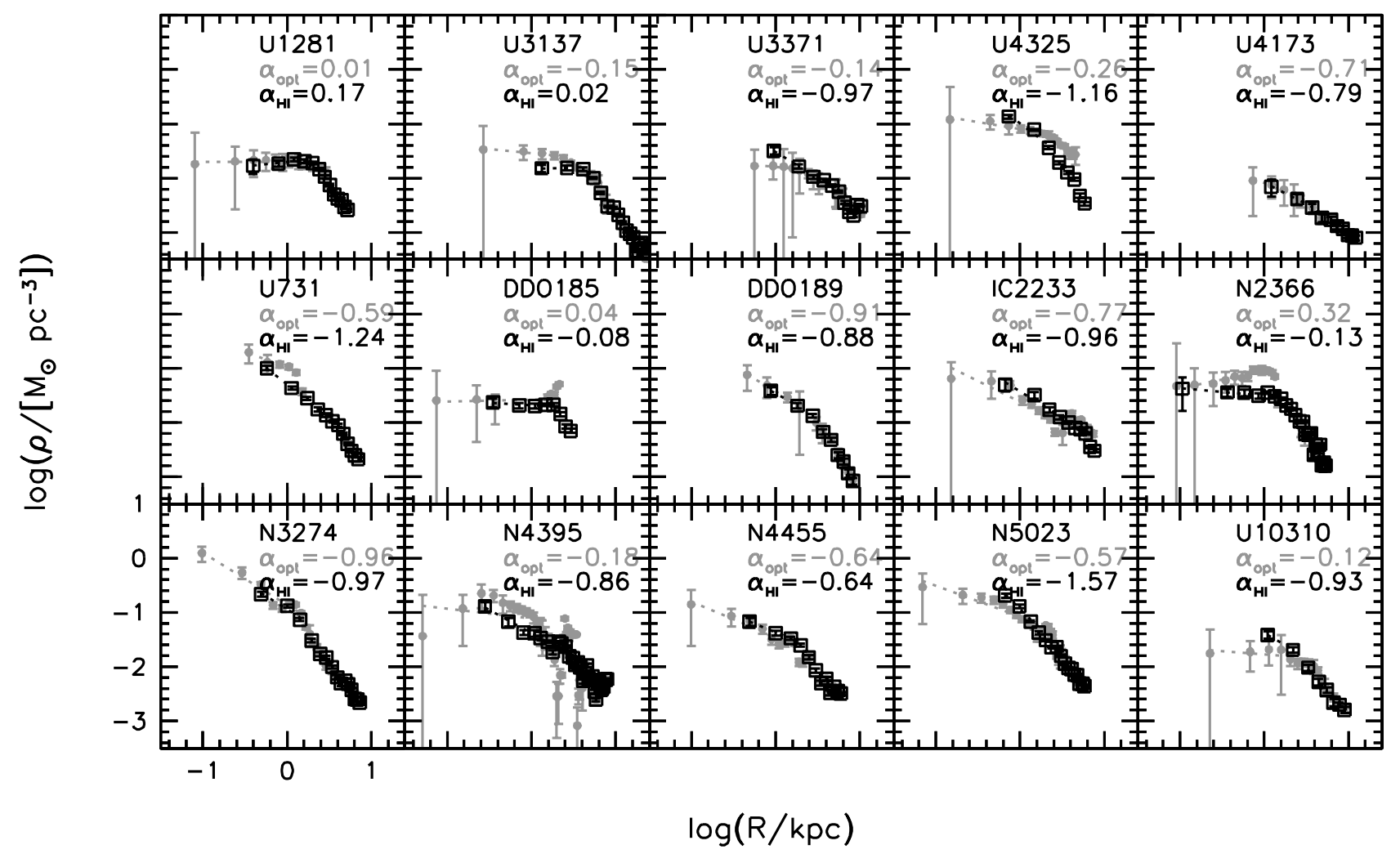

Fig. 16. Comparison of mass density profiles for a number of galaxies as derived from the high-resolution curves (grey data points) and from medium resolution Hi curves (black points). The dashed lines indicate the range over which the inner slope is fitted. Derived values are indicated in each panel.

unambiguously prove that a galaxy has a bona-fide NFW halo and is inconsistent with the pseudo-isothermal halo model, it needs to have a steep inner slope, and a small value of $r_{\text {in }}$ and a low surface brightness. Such galaxies have not been found yet.

\subsubsection{Resolution and slope}

One of the conclusions of $\mathrm{dBMBR}$ is that as the resolution of rotation curves ) is increased (i.e. $r_{\text {in }}$ is decreased), the derived inner slope should become shallower. We test this by comparing the slopes and inner radii for a number of curves for which we have high-resolution curves and medium-resolution $\left(\sim 15^{\prime \prime}\right)$ Hi curves. We determined the slopes of both the high-resolution curves and the HI curves.

In Fig. 16 we plot the inversions of the high-resolution hybrid curves and the lower-resolution Hi curves. The slopes as derived from these curves are shown in the figure. Those derived from the Hi curves tend to be steeper than those from the equivalent high-resolution curves. The values of the slopes in Fig. 16 are slightly different from those given in Fig. 13. This is because the Hi profiles generally did not extend as far inward as the hybrid profiles, and we had to choose a larger upper fitting radius. In Fig. 17 we plot the positions that the high-resolution and Hi curves occupy in the $\alpha-r_{\text {in }}$ diagram. The general trend is indeed that as the resolution is increased, the

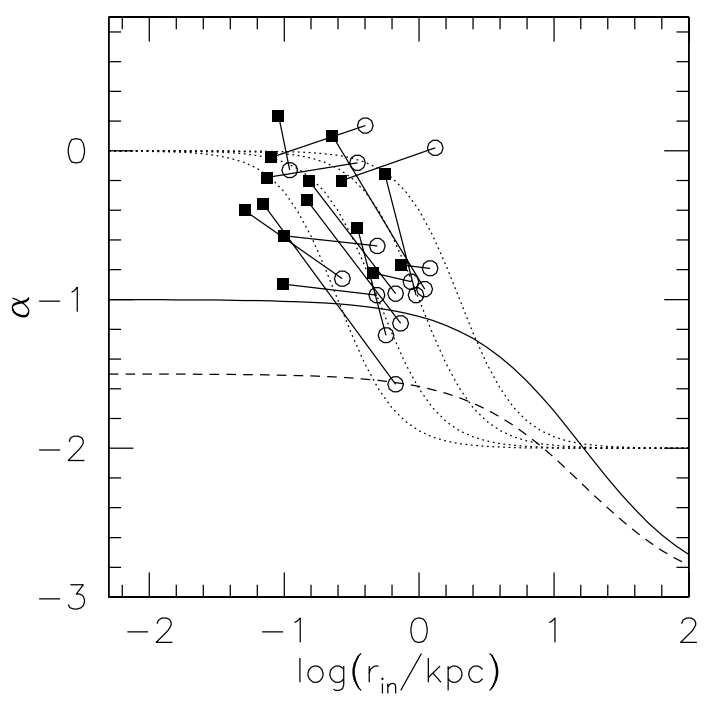

Fig. 17. Comparison of the inner slopes $\alpha$ and innermost radii $r_{\text {in }}$ of the high-resolution curves and the medium resolution $\mathrm{HI}$ curves shown in Fig. 16. The filled squares indicate the values derived from the high-resolution curves; open circles indicate values derived from the Hi curves. Corresponding pairs are connected. Galaxies move along the isothermal halo curves when resolution is increased.

galaxies move towards smaller slopes, more or less along the pseudo-isothermal halo model curves. 
It is interesting to compare our analysis with that of van den Bosch \& Swaters (2001) (hereafter vdBS), who investigated the Hi curves of a number of galaxies for which we now have high-resolution data. Their conclusion was that the majority of the galaxies they investigated was consistent with a $\Lambda$ CDM scenario, but they also noted that it is difficult to distinguish between a cusp and a core model at the typical resolution of the Hi data. It is clear that this was because the lower spatial and velocity resolution makes it possible to force a NFW fit on the data, even though it is not always of the shape preferred by the data. We will discuss the galaxies they consider in turn.

U731: The Hi curve differs substantially from the high-resolution curve. Though our NFW fit gives similar parameters to the vdBS fit $(c \sim 18$ versus their $c \sim 16)$, our curve provides a much inferior fit. This is mainly caused by the more linear inner curve. The inner slope of the high-resolution mass density profile shows a large change away from the optimum CDM value.

U3371: The high-resolution curve is more linear and rises less steeply than the Hi curve. The slope of the inner curve again shows a large change: the HI value is $\alpha \sim 1$ and consistent with CDM, the new value is $\alpha \sim 0$ and consistent with a constant-density core. We were unable to derive a sensible NFW model fit.

U4325: This galaxy shows the same behaviour as U3371: the slope derived from the HI data is optimum for CDM, whereas the slope derived from the high-resolution data is consistent with a constant density core.

$\mathbf{N 4 3 9 5}$ (U7524): This is the best-resolved HI curve in the vdBS sample, but here we also see the same trends noted above: the slope of the Hi curve is steeper than the slope of the high-resolution curve. The new curve also differs markedly from the Hi curve. The difference between NFW and pseudo-isothermal is most marked in the inner parts. The increase in resolution has made this galaxy less consistent with CDM.

N4455 (U7603): vdBS note that for this galaxy no meaningful CDM fit could be derived. We find identical slopes for both sets of data. As a note of interest, we note that the NFW fit to the Hi curve presented in vdBS shows the same systematic residuals as the $\mathrm{H} \alpha$ LSB rotation curves presented in dBMR.

DDO189 (U9211): We find similar slopes as vdBS and also the models are of similar quality. The new data does not prefer one model over the other.

Of the 6 galaxies in this small subsample vdBS find that 5 are consistent with CDM, with one dubious case. The increased resolution suggests that only one of these 6 galaxies (DDO189) is still consistent with CDM (this is also the galaxy with the poorest high-resolution data in the subsample). For two galaxies CDM is perhaps inconsistent with the data (U731 and N4455), whereas for the remaining three the data are clearly inconsistent with the CDM models. In summary, we conclude that the slopes of rotation curves are best described by a pseudo-isothermal halo model.

\section{Conclusions}

We have presented high-resolution $\mathrm{H} \alpha / \mathrm{HI}$ rotation curves of a sample of $26 \mathrm{LSB}$ and dwarf galaxies. We have fitted mass-models to these rotation curves assuming both a pseudo-isothermal (core-dominated) halo and a CDM NFW (cusp-dominated) halo. We find that the pseudoisothermal halos generally provide better fits, though the difference is maybe not as pronounced as in $\mathrm{dBMR}$, which can be traced back to the fact that our sample contains more galaxies of higher surface brightness than the dBMR sample.

We find more galaxies with low concentration parameters than predicted by numerical CDM simulations. An analysis of the mass-density profiles of the halos, as derived from minimum-disk rotation curves, shows that the galaxies in our sample are dominated by more-or-less constant density cores. As shown in dBMBR the few galaxies that show slopes consistent with CDM are usually the ones that are not well-resolved, so that one traces the edges of the constant-density cores rather than the cores themselves. We have illustrated this explicitly by comparing the mass-density profiles of a number of galaxies for which we have both high-resolution $\mathrm{H} \alpha$ curves and medium-resolution Hi curves. In general the slopes derived from the high-resolution curves are less steep than those from the Hi curves.

In conclusion, our high resolution rotation data on nearby dwarfs and LSB galaxies show that the halos of late-type LSB and dwarf galaxies are dominated by kpcsized constant density cores inconsistent with the predictions of cuspy dark matter halos in cosmological numerical simulations.

Acknowledgements. EdB would like to thank ANSTO for their financial support which made attending the February 2001 observing run possible. AB thanks the Programme National Galaxies for financial support of his observing runs at the Observatoire de Haute Provence. We thank Stacy McGaugh for useful discussions.

\section{References}

Begeman, K. G. 1987, Ph.D. Thesis, University of Groningen Bell, E. F., \& de Jong, R. S. 2001, ApJ, 550, 212

Blais-Ouellette, S., Amram, P., \& Carignan, C. 2001, AJ, 121, 1952

Bolatto, A. D., Simon, J. D., Leroy, A., \& Blitz, L. 2002, ApJ, 565,238

Borriello, A., \& Salucci, P. 2001, MNRAS, 323, 285

Bosma, A. 1978, Ph.D. Thesis, University of Groningen

Bosma, A. 1999, in Galaxy Dynamics, ed. D. R. Merritt, M. Valluri, \& J. A. Sellwood, ASP Conf. Ser., 182 (San Francisco: ASP), 339

Bosma, A., Byun, Y. I., Freeman, K. C., \& Athanassoula, E. 1992, ApJ, 400, L23

Bottema, R. 1997, A\&A, 328, 517

Broeils, A. H. 1992, A\&A, 256, 19

Bullock, J. S., Kolatt, T. S., Sigad, Y., et al. 2001, MNRAS, 321,559 
Corradi, R. L. M., Boulesteix, J., Bosma, A., Amram, P., \& Capaccioli, M. 1991, A\&A, 244, 27

de Blok, W. J. G., van der Hulst, J. M., \& Bothun, G. D. 1995, MNRAS, 274, 235

de Blok, W. J. G., McGaugh, S. S., \& van der Hulst, J. M. 1996, MNRAS, 283, 18

de Blok, W. J. G., \& McGaugh, S. S. 1997, MNRAS, 290, 533

de Blok, W. J. G., McGaugh, S. S., \& Rubin, V. C. 2001a, AJ, 122,2396

de Blok, W. J. G., McGaugh, S. S., Bosma, A., \& Rubin, V. C. 2001b, ApJ, 552, L23 (dBMBR)

de Jong, R. S. 1996, Ph.D. Thesis, University of Groningen

Hopp, U., \& Schulte-Ladbeck, R. E. 1991, A\&A, 248, 1

Hunter, D. A., Elmegreen, B. G., \& van Woerden, H. 2001, preprint [astro-ph/0104091]

Jing, Y. P. 2000, ApJ, 535, 30

Karachentsev, I. D., Karachentseva, V. E., \& Parnovskij, S. L. 1993, Astr. Nachr., 314, 97

Kurtz, M. J., \& Mink, D. J. 1998, PASP, 110, 934

Lemaître, G., Kohler, D., Lacroix, D., Meunier, J. P., \& Vin, A. 1990, A\&A, 228, 546

Loader, C. 1999, Local Regression and Likelihood (SpringerVerlag: New York)

Makarova, L. 1999, A\&AS, 139, 491

McGaugh, S. S., \& Rubin, V. C., \& de Blok, W. J. G. 2001, AJ, 122, 2381

Moore, B. 1994, Nature, 370, 629

Moore, B., Quinn, T., Governato, F., Stadel, J., \& Lake, G. 1999, MNRAS, 310, 1147

Navarro, J. F., Frenk, C. S., \& White, S. D. M. 1996, ApJ, 462, 563

Navarro, J. F., Frenk, C. S., \& White, S. D. M. 1997, ApJ, 490, 493
Pickering, T. E., Navarro, J. F., Rix, H.-W., \& Impey, C. D. 1998, in Galactic Halos, ed. D. Zaritsky, ASP, 136, 199

Rubin, V. C., Boss, A. P., Ford, W. K., Jr., \& Kenney, J. D. 1989, AJ, 98, 1246

Salucci, P., \& Burkert, A. 2000, ApJ, 537, L9

Salucci, P. 2001, MNRAS, 320, L1

Sellwood, J. A. 1999, in Galaxy Dynamics, ed. D. R. Merritt, M. Valluri, \& J. A. Sellwood, ASP Conf. Ser., 182 (San Francisco: ASP), 351

Stil, J. 1999, Ph.D. Thesis, University of Leiden

Swaters, R. A. 1999, Ph.D. Thesis, University of Groningen

Swaters, R. A., Schoenmakers, R. H. M., Sancisi, R., \& van Albada, T. S. 1999, MNRAS, 304, 330

Swaters, R. A., Madore, B. F., \& Trewhella, M. 2000, ApJ, 531, L107

Swaters, R. A., van Albada, T. S., van der Hulst, J. M., \& Sancisi, R. 2002, A\&A, in press

Swaters, R. A., \& Balcells, M. 2002, A\&A, submitted

van Albada, T. S., \& Sancisi, R. 1986, Phil. Trans. R. Soc. Lond. A, 320, 447

van den Bosch, F., \& Swaters, R. A. 2001, MNRAS, 325, 1017

van den Hoek, L. B., de Blok, W. J. G., van der Hulst, J. M., \& de Jong, T. 2000, A\&A, 357, 397

van der Hulst, J. M., Skillman, E. D., Smith, T. R., et al. 1993, AJ, 106, 548

van der Kruit, P. C., \& Searle, L. 1981, A\&A, 95, 105

Van der Kruit, P. C., \& Bosma, A. 1978, A\&AS, 34, 259

Verheijen, M. A. W. 1997, Ph.D. Thesis, University of Groningen

Walter, F., \& Brinks, E. 2001, AJ, 121, 3026 